\title{
Effects of High Intensity Exercise on Oxidative Stress and Antioxidant Status in Untrained Humans: A Systematic Review
}

\author{
Yining Lu ${ }^{1}$, Huw D. Wiltshire ${ }^{1}$, Julien S. Baker ${ }^{2}{ }^{\mathbb{D}}$ and Qiaojun Wang ${ }^{3, *}$ \\ 1 Cardiff School of Sport and Health Sciences, Cardiff Metropolitan University, Cardiff CF5 2YB, UK; \\ st20184530@outlook.cardiffmet.ac.uk (Y.L.); hwiltshire@cardiffmet.ac.uk (H.D.W.) \\ 2 Department of Sport, Physical Education and Health, Hong Kong Baptist University, \\ Kowloon Tong, Hong Kong; jsbaker@hkbu.edu.hk \\ 3 Faculty of Sport Science, Ningbo University, Ningbo 315000, China \\ * Correspondence: wangqiaojun@nbu.edu.cn; Tel.: +86-13805885586
}

check for updates

Citation: Lu, Y.; Wiltshire, H.D.; Baker, J.S.; Wang, Q. Effects of High Intensity Exercise on Oxidative Stress and Antioxidant Status in Untrained Humans: A Systematic Review. Biology 2021, 10, 1272. https:/ / doi.org/10.3390/biology10121272

Academic Editor: Redha Taiar

Received: 31 October 2021

Accepted: 2 December 2021

Published: 4 December 2021

Publisher's Note: MDPI stays neutral with regard to jurisdictional claims in published maps and institutional affiliations.

Copyright: (c) 2021 by the authors. Licensee MDPI, Basel, Switzerland. This article is an open access article distributed under the terms and conditions of the Creative Commons Attribution (CC BY) license (https:// creativecommons.org/licenses/by/ $4.0 /)$.
Simple Summary: This systematic review aims to investigate the influence of HIE on oxidative stress and antioxidant status in untrained humans. Following the PRISMA selection process, 21 studies were finally included. A rigorous methodological quality assessment (MQA) and levels of evidence was applied. There was strong evidence that acute oxidative stress occurs at the cessation of HIE when compared to resting states. The HIE-induced oxidative stress is transient and recoverable due to the stimulated endogenous antioxidant system. While the responses of antioxidant systems are lagging and lasting, multiple measurement times are suggested. Higher fitness levels are associated with less oxidative stress and regular physical exercise appears to improve antioxidant capacity and provide health benefits.

Abstract: Participation in exercise promotes health. High intensity exercise (HIE) has become increasingly popular among the general population, however, its effects on exercise-induced oxidative stress and antioxidant status in untrained humans is not clear. The aim of this systematic review was to investigate the influence of HIE on oxidative stress and antioxidant status in untrained humans. Web of Science, PubMed, MEDLINE, and Scopus were searched until March 2021. A methodological quality assessment valuation/estimation was additionally carried out in the final sample of studies. Following the PRISMA selection process, 21 studies were finally included. There was strong evidence that acute oxidative stress following the cessation of HIE exists when compared to resting states. The HIE-induced oxidative stress is transient and is most likely restored to normal levels within $24 \mathrm{~h}$ due to the stimulated endogenous antioxidant system whose response was lagging and lasting. Physically active humans had better antioxidant systems and suffered less oxidative stress after HIE. A physically active lifestyle was considered to enhance antioxidant capacity. For untrained humans, $\mathrm{HIE}$ with intensities above $70 \% \mathrm{VO}_{2}$ max are proposed for initial exercise levels based on the findings reported here.

Keywords: high intensity exercise; oxidative stress; antioxidant; untrained

\section{Introduction}

Free radicals are rogue molecules that damage cells. Denham Harman (1956) first discovered the active properties of free radicals and suggested the free radical theory of aging [1]. The theory proposed that the production of free radicals, such as reactive oxygen species (ROS), is inevitable during metabolism. ROS are active substances containing oxygen occurring in the human body or the natural environment. Normal metabolism in the body can produce ROS, which can initiate the formation of free radicals [2]. However, any uncontrolled production of reactive oxygen species can lead to oxidative damage to proteins, DNA, and lipids [3,4]. 
Antioxidants are substances that minimize the harmful effects of oxygen. These substances help trap and neutralize free radicals, thereby preventing damage on the human body. The antioxidant system in the human body consists of antioxidant metabolites and enzymes that impede the production of ROS by removing these active substances before they can cause damage to the important components of cells [5]. However, ROS are not always harmful, and physiologically, appropriate concentrations of ROS can promote immunity [6]. Therefore, the role of the body's antioxidant system is not to remove ROS completely, but to control them at appropriate levels.

Physiologically, antioxidants and oxidants are in equilibrium. The body's endogenous antioxidant defense system (non-enzymatic and enzymatic), under normal conditions, is effective against the potentially harmful effects of ROS [6,7]. When oxidizing substances increase, oxidative stress occurs. Increasing evidence shows that most health problems and diseases caused by aging are related to endogenous ROS production and oxidative stress [8]. It is widely believed that most age-related health problems, ranging from wrinkles, and including cardiovascular disease, cancer, and Alzheimer's disease, are linked to excessive oxidative stress [9-11].

Many studies have shown that with the increase in oxygen consumption during exercise, the production of ROS increases [5,12]. When the ability of the antioxidant system is insufficient to counterbalance ROS production during exercise, oxidative stress occurs. Davies et al. (1982) used electron spin resonance (ESR) for the first time to directly confirm the significant increase of free radicals in muscles following exercise [13]. Over the past 40 years, various studies have emerged investigating the effects of oxidative stress induced by exercise.

Studies have also indicated that regular exercise can upregulate the body's antioxidant system and increase its resistance to oxidative stress [14-17]. Regular exercise is beneficial to health, and it can reduce the risk of cancer, cardiovascular disease, diabetes, and other chronic diseases $[2,8,18-20]$.

Despite this, Davies et al. (1982) and Jackson et al. (1985) used ESR to provide direct evidence that exercise may induce oxidative stress $[13,21]$. The elevation of biomarkers of oxidative damage in the blood and skeletal muscle also provides indirect evidence for oxidative stress induced by exercise. During exercise, skeletal muscle contractions produce free radicals, while increased oxygen consumption produces a large amount of ROS [22-25]. If the body's antioxidant defense is insufficient, cells and tissues will suffer oxidative damage [26].

Physical exercise is a complex biological activity that constantly challenges the oxidationantioxidant balance of the body (cells, tissues, organs, etc.) while maintaining biological balance [27]. The adjustment of exercise on oxidative stress can be acute or long-term. Acute adjustment is an incomplete adaptation that can easily lead to oxidative damage, so it is important to give the body sufficient rest following exercise to restore balance. The process of balancing-breaking the balance-restoring the balance helps improve the body's ability to cope with oxidative stress. In fact, regular exercise can fundamentally upregulate the body's endogenous antioxidant system [28]. Moderate aerobic exercise is often used to improve the body's antioxidant capacity and reduce chronic diseases. At the same time, more and more studies suggest that high intensity exercise (HIE) may be more effective in promoting fitness and health than traditional continuous training [29-31].

Due to its efficient timesaving protocols and effectiveness, HIE has been the subject of more and more attention in recent years among athletes, bodybuilders, and individuals with chronic diseases. However, along with the increasing interest in HIE, there are questions that need consideration. One such question relates to the relationship between HIE and oxidative stress. This question may directly affect the arrangement of athletic training loads, the choice of fitness methods, and the safety of exercise for patients with chronic diseases.

To date, effects of HIE on oxidative stress in untrained humans are inconclusive. Therefore, this systematic review aims to systematically analyze effects of a single bout of 
HIE on oxidative stress markers and antioxidant status in untrained humans. A further aim was to investigate if long-term HIE can influence exercise-induced oxidative stress and upgrade the antioxidant system, and furthermore, to provide important information for physically inactive individuals to participant in HIE.

\section{Methods}

\subsection{Data Sources and Searches}

According to the Preferred Reporting Items for Systematic reviews and meta-analysis (PRISMA), a systematic literature search, limited to literature published in English and Chinese, was conducted in March 2021 using four electronic databases (PubMed, MEDLINE, Web of Science, SCOPUS). Following this process, the literature list obtained was then manually searched and the results were placed in Endnote (Endnote 20, Clarivate, Boston, MA, USA).

Search terms were limited to titles and abstracts and based on all possible combinations of the following keywords: high-intensity, interval, high-intensity interval, exhaustive, acute, training, exercise, exercised-induced, physical activity, oxidative stress, damage, oxidative damage.

\subsection{Inclusion Criteria}

\subsubsection{Type of Study}

Studies involving high-intensity exercise protocols targeted at exploring the effects on oxidative stress markers and antioxidant status were included. Exercise protocols with a principal focus on high-intensity, high-intensity interval/intermittent, sprint, maximal, exhaustive, acute are considered as HIE in this review.

\subsubsection{Type of Participants}

Studies conducted in healthy untrained humans were included. No gender constraints were applied to all participants without disability and obesity. Participants under 16 years old were not included. Studies that used HIE as a treatment for specific illnesses were excluded and so were animal studies. Participants were considered as untrained when participants were described as physically inactive, sedentary, non-athletes, recreationally active, and physically active.

\subsubsection{Type of Protocols}

The inclusion criteria for studies in the review was as follows: (a) at least one bout of training/exercise was carried out; (b) oxidative stress markers were measured at baseline and post-training.

High intensity can be broadly defined as an intensity that is greater than that of exercise performed at a level corresponding to the anaerobic threshold. In this review, protocols were defined as high-intensity if: (a) the participant performed with an "all-out" effort [32]; (b) protocols were described as "maximal", "sprint", or "high"; (c) the intensity was $\geq 70 \%$ maximal oxygen uptake $\left(\mathrm{VO}_{2} \mathrm{max}\right)$; (d) the participants' heart rates were $\geq 70 \%$ of their maximal heart rate. (e) There were no restrictions applied regarding the mode and the duration of the protocol.

\subsubsection{Type of Outcomes}

Outcomes included oxidative markers (directly detected by electron spin resonance (ESR) and indirectly measured by malondialdehyde (MDA) and thiobarbituric acid reactive substances (TBARS)), antioxidant enzyme activities (superoxide dismutase (SOD), glutathione peroxidase (GPX), catalase (CAT) and glutathione (GSH)), and total antioxidant capacity (TAC).

Articles that satisfied the above criteria were included in the review. Meanwhile, articles were excluded if (a) they were published after March 2021; (b) full text of the articles was not found; (c) articles were not written in English or Chinese; (d) studies that 
used a different intervention (e.g., drugs or diet) that may have impacted on oxidative stress were excluded. When the same data were presented in multiple publications, the first published study was used for the review and analysis.

\subsection{Identification of Eligible Studies}

Eligible studies were empirical studies conducted in untrained humans that measured oxidative markers and antioxidant enzyme activities after a single bout of HIE or a longterm HIE protocol. Two authors (Yining $\mathrm{Lu}$ and Qiaojun Wang) were responsible for retrieving selected articles from four databases and applying inclusion and exclusion criteria to determine eligible studies. The articles were then carefully read and evaluated by a further two independent authors (Huw Wiltshire and Julien Baker) to determine whether they should be included.

\subsection{Quality Assessment}

The results were analyzed using methodological quality assessment (MQA) according to the revised Downs \& Black Quality Index (1998) (Appendix A) [33]. The MQA was implemented by two authors (Yining Lu and Qiaojun Wang) and were proofread by the other authors. Finally, a consultation session was arranged to reconcile any differences. The revised edition contained a total of 10 questions; 5 of the questions assessed report quality, 4 assessed internal validity, and 1 assessed power. A "yes" or "no" for each question was recorded as a 1 or 0 , respectively. The total score was 10 . Studies were defined as high quality if they scored an overall score of 7 or higher. Studies were defined as low quality if they received a total score of 5 or 6 , and studies were defined as very low quality when they obtained a score under 4 [34].

\subsection{Level of Evidence}

The levels of evidence were divided into three levels. Evidence was strong when three or more high-quality studies indicated consistent findings. The evidence was considered moderate when two high-quality studies showed consistent results. The evidence was limited when it was based on low-quality studies or a single study [34].

\subsection{Data Extraction}

The data included in the study were extracted in several structured table formats covering the following topics: sociodemographic characteristics of participants (age, gender, weight, body mass index, maximal oxygen uptake, diet, lifestyle, socio-economic level, tobacco, and alcohol), exercise protocol (specified as modality, type of protocol, No. of bouts, duration of bouts, duration of protocols, work/rest ratio, intensity), training protocols (specified as duration, frequency, No. of bouts, duration of bouts, intensity, duration of recovery), selected biomarkers, and findings. All studies measured the baseline status of the subjects. Some studies measured oxidative damage and antioxidant status at only one time-point (TP), mostly at the cessation of exercise, while others included multiple post-exercise measures following exercise completion.

\section{Results}

\subsection{Search Results}

The selection process and the number of articles identified at each step are shown in Figure 1. Eight thousand three hundred and fifty records (8350) were retrieved in the initial database search. Nine hundred and seven (907) duplicates were removed. After title and abstract screening, ninety-six (96) records were reserved for eligibility assessment. Seventy-three (75) articles were excluded after full-text examination (excluded reasons detailed in Figure 1). The most common reason for exclusion was that the participants were not human. Finally, twenty-one (21) were included in the present review. 


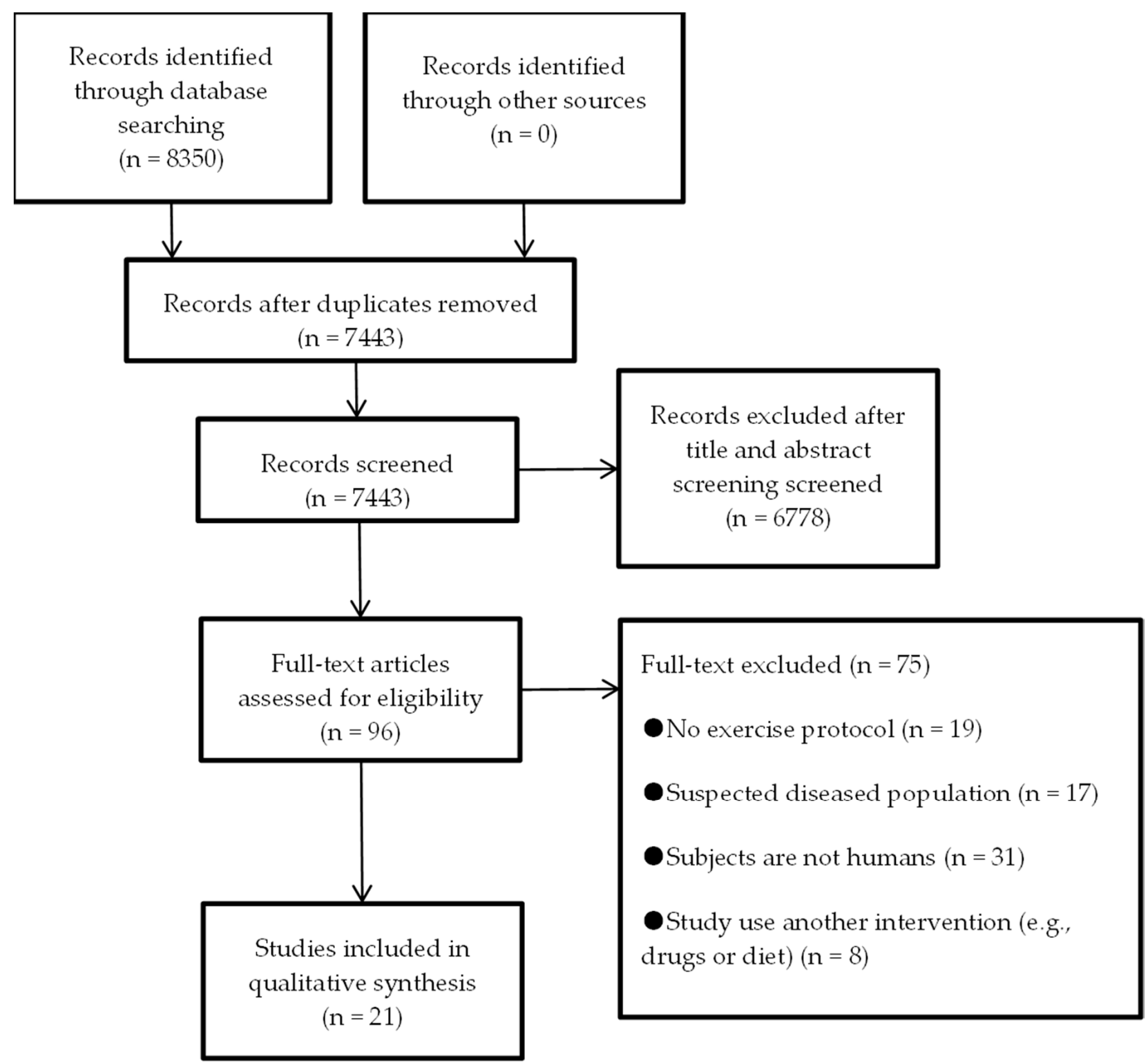

Figure 1. PRISMA flow diagram displaying the selection process.

\subsection{Methodological Quality Assessment}

The MQA scoring results of the selected 21 manuscripts are shown in Table 1 . The total quality scores of the papers are shown as a percentage value in the last column. The quality of manuscripts ranged from $60 \%$ to $80 \%$, and the average quality index was $69 \%$. Twelve studies were high-quality (score $\geq 7$ ), nine studies were low-quality ( $\leq$ score $\leq 6)$, and no study was defined with a very low quality (score $\leq 4$ ).

All manuscripts specified objectives $(21 / 21)$, characteristics of the participants $(21 / 21)$, findings (21/21), use of statistical tests $(21 / 21)$, and a significance level of $p<0.05(21 / 21)$. In addition, 11 of the 21 studies explicitly mentioned the intervention, and 8 of the 21 studies were randomized. Finally, none of studies reported adverse events because of the interventions. 
Table 1. Methodological quality assessment.

\begin{tabular}{|c|c|c|c|c|c|c|c|c|c|c|c|}
\hline Reference & Q1 & Q2 & Q3 & Q4 & Q5 & Q6 & Q7 & Q8 & Q9 & Q10 & Total \\
\hline Ammar et al., 2020 [35] & 1 & 1 & 1 & 1 & 0 & 0 & 1 & 1 & 1 & 1 & $80 \%$ \\
\hline Baker et al., 2004 [36] & 1 & 1 & 1 & 1 & 0 & 0 & 1 & 1 & 1 & 1 & $80 \%$ \\
\hline Berzosa er al., 2011 [37] & 1 & 1 & 1 & 1 & 0 & 0 & 1 & 1 & 1 & 1 & $80 \%$ \\
\hline Bogdanis et al., 2013 [38] & 1 & 1 & 1 & 1 & 0 & 0 & 1 & 0 & 0 & 1 & $60 \%$ \\
\hline Bouzid et al., 2014 [39] & 1 & 1 & 1 & 1 & 0 & 0 & 1 & 1 & 0 & 1 & $70 \%$ \\
\hline Djordjevic et al., 2012 [40] & 1 & 1 & 1 & 1 & 0 & 0 & 1 & 1 & 0 & 1 & $70 \%$ \\
\hline Falone et al., 2010 [41] & 1 & 1 & 1 & 1 & 0 & 0 & 1 & 1 & 0 & 1 & $70 \%$ \\
\hline Finkler et al., 2016 [42] & 1 & 1 & 1 & 1 & 0 & 0 & 1 & 0 & 0 & 1 & $60 \%$ \\
\hline Fisher et al., 2011 [43] & 1 & 1 & 1 & 1 & 0 & 0 & 1 & 0 & 0 & 1 & $60 \%$ \\
\hline Groussard et al., 2003 [44] & 1 & 1 & 1 & 1 & 0 & 0 & 1 & 0 & 0 & 1 & $60 \%$ \\
\hline Hajizadeh et al., 2017 [45] & 1 & 1 & 1 & 1 & 0 & 0 & 1 & 1 & 1 & 1 & $80 \%$ \\
\hline Jammes et al., 2004 [46] & 1 & 1 & 1 & 1 & 0 & 0 & 1 & 0 & 0 & 1 & $60 \%$ \\
\hline Jamurtas et al., 2018 [47] & 1 & 1 & 1 & 1 & 0 & 0 & 1 & 1 & 1 & 1 & $80 \%$ \\
\hline Kyparos et al., 2007 [48] & 1 & 1 & 1 & 1 & 0 & 0 & 1 & 0 & 1 & 1 & $70 \%$ \\
\hline Miyazaki et al., 2001 [49] & 1 & 1 & 1 & 1 & 0 & 0 & 1 & 1 & 0 & 1 & $70 \%$ \\
\hline Parker et al., 2014 [50] & 1 & 1 & 1 & 1 & 0 & 0 & 1 & 0 & 0 & 1 & $60 \%$ \\
\hline Parker et al., 2018 [51] & 1 & 1 & 1 & 1 & 0 & 0 & 1 & 1 & 1 & 1 & $80 \%$ \\
\hline Seifi-Skishahr et al., 2008 [52] & 1 & 1 & 1 & 1 & 0 & 0 & 1 & 0 & 0 & 1 & $60 \%$ \\
\hline Steinberg et al., 2007 [53] & 1 & 1 & 1 & 1 & 0 & 0 & 1 & 0 & 0 & 1 & $60 \%$ \\
\hline Wadley et al., 2016 [54] & 1 & 1 & 1 & 1 & 0 & 0 & 1 & 1 & 1 & 1 & $80 \%$ \\
\hline \multirow[t]{2}{*}{ Wiecek et al., 2018 [55] } & 1 & 1 & 1 & 1 & 0 & 0 & 1 & 0 & 0 & 1 & $60 \%$ \\
\hline & \multicolumn{5}{|c|}{ Reporting } & $\begin{array}{r}\text { Int } \\
\text { validit }\end{array}$ & nal & \multicolumn{2}{|c|}{$\begin{array}{c}\text { Internal } \\
\text { validity- } \\
\text { confounding }\end{array}$} & Power & Average \\
\hline TOTAL/21 & 21 & 21 & 21 & 21 & 0 & 0 & 21 & 11 & 8 & 21 & $69 \%$ \\
\hline
\end{tabular}

\subsection{Type of Studies}

Details of exercise testing and training regimes of the included studies are outlined in Table 2. Exercise testing involved exercise protocols used for analysis of the acute changes in oxidative stress and antioxidant status, while training were protocols used to investigate the effect of long-term exercise on oxidative stress and antioxidant capacity. Thus, 15/21 studies investigated acute oxidative response following a single bout of HIE at TP0 (Table 3 details corresponding TPs and findings for each study); 13/21 studies included multiple post-exercise measures after exercise; $3 / 21$ studies compared HIE-induced oxidative stress and antioxidant status pre and post high intensity training; $2 / 21$ studies measured oxidative damage between untrained participants and others with different physical activity characteristics; 8/21 studies compare oxidative stress following different types of HIE. 
Table 2. Details of exercise testing and training of the included studies.

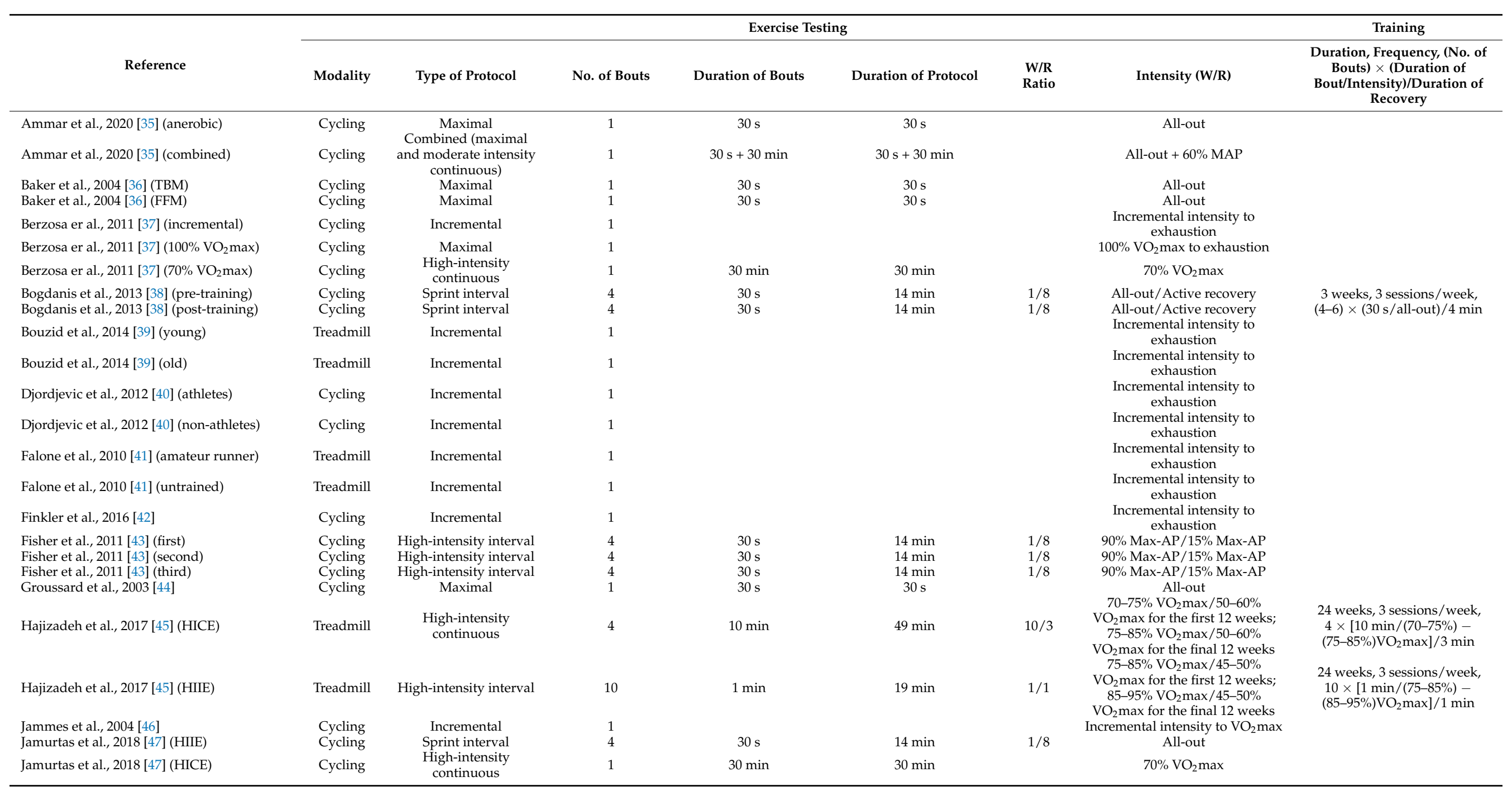


Table 2. Cont.

\begin{tabular}{|c|c|c|c|c|c|c|c|c|}
\hline \multirow[b]{2}{*}{ Reference } & \multicolumn{7}{|c|}{ Exercise Testing } & \multirow{2}{*}{$\begin{array}{c}\text { Training } \\
\begin{array}{c}\text { Duration, Frequency, (No. } \\
\text { of Bouts) } \times(\text { Duration of } \\
\text { Bout/Intensity)/Duration } \\
\text { of Recovery }\end{array} \\
\end{array}$} \\
\hline & Modality & Type of Protocol & No. of Bouts & Duration of Bouts & Duration of Protocol & $\begin{array}{l}\text { W/R } \\
\text { Ratio }\end{array}$ & Intensity $(W / R)$ & \\
\hline Kyparos et al., 2007 [48] & $\begin{array}{l}\text { Shuttle } \\
\text { run }\end{array}$ & Maximal & 1 & & & & All-out & \multirow{12}{*}{$\begin{array}{l}12 \text { weeks, } 5 \text { sessions } / \text { week } \\
1 \times\left(60 \mathrm{~min} / 80 \% \mathrm{VO}_{2} \max \right)\end{array}$} \\
\hline Miyazaki et al., 2001 [49] (pre-training) & Cycling & Incremental & 1 & & & & $\begin{array}{c}\text { Incremental intensity to } \\
\text { exhaustion }\end{array}$ & \\
\hline Miyazaki et al., 2001 [49] (post-training) & Cycling & Incremental & 1 & & & & $\begin{array}{c}\text { Incremental intensity to } \\
\text { exhaustion }\end{array}$ & \\
\hline Parker et al., 2014 [50] $\left(70 \% \mathrm{VO}_{2} \max \right)$ & Cycling & $\begin{array}{l}\text { High-intensity } \\
\text { interval }\end{array}$ & 1 & $5 \mathrm{~min}$ & $5 \mathrm{~min}$ & $5 / 12$ & $\begin{array}{c}70 \% \mathrm{VO}_{2} \max / \text { passive seated } \\
\text { rest }\end{array}$ & \\
\hline Parker et al., 2014 [50] $\left(85 \% \mathrm{VO}_{2} \max \right)$ & Cycling & $\begin{array}{l}\text { High-intensity } \\
\text { interval }\end{array}$ & 1 & $5 \mathrm{~min}$ & $5 \mathrm{~min}$ & $5 / 12$ & $\begin{array}{c}85 \% \mathrm{VO}_{2} \max / \text { passive seated } \\
\text { rest }\end{array}$ & \\
\hline Parker et al., 2014 [50] $\left(100 \% \mathrm{VO}_{2} \max \right)$ & Cycling & $\begin{array}{l}\text { High-intensity } \\
\text { interval }\end{array}$ & 1 & $5 \mathrm{~min}$ & $5 \mathrm{~min}$ & $5 / 12$ & $\begin{array}{c}100 \% \mathrm{VO}_{2} \text { max } / \text { passive } \\
\text { seated rest }\end{array}$ & \\
\hline Parker et al., 2018 [51] (HIIE) & Cycling & $\begin{array}{l}\text { High-intensity } \\
\text { interval }\end{array}$ & 5 & $4 \mathrm{~min}$ & $24 \min$ & $4 / 1$ & $75 \% W \max$ & \\
\hline Parker et al., 2018 [51] (SIE) & Cycling & Sprint interval & 4 & $30 \mathrm{~s}$ & $15.5 \mathrm{~min}$ & $1 / 9$ & All-out & \\
\hline Seifi-Skishahr et al., 2008 [52] & Treadmill & $\begin{array}{l}\text { High-intensity } \\
\text { continuous }\end{array}$ & 1 & $30 \mathrm{~min}$ & $30 \mathrm{~min}$ & & $75 \% \mathrm{VO}_{2} \max$ & \\
\hline Wadley et al., 2016 [54] (LV-HIIE) & Cycling & $\begin{array}{l}\text { High-intensity } \\
\text { interval }\end{array}$ & 10 & $1 \mathrm{~min}$ & $19 \min$ & $1 / 1$ & $90 \% \mathrm{VO}_{2} \max$ & \\
\hline Wadley et al., 2016 [54] (HICE) & Cycling & $\begin{array}{l}\text { High-intensity } \\
\text { continuous }\end{array}$ & 1 & $20 \mathrm{~min}$ & $20 \mathrm{~min}$ & & $80 \% \mathrm{VO}_{2} \max$ & \\
\hline Wiecek et al., 2018 [55] & Cycling & Maximal & 1 & $20 \mathrm{~s}$ & $20 \mathrm{~s}$ & & All-out & \\
\hline
\end{tabular}

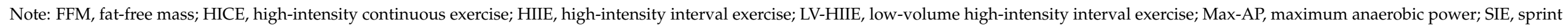
interval exercise; $\mathrm{VO}_{2} \mathrm{max}$, maximal oxygen uptake; All-out: encompasses intensities described by the authors as "sprints", "maximal", "maximal exercise", "exhaustion", "near the $\mathrm{VO}_{2}$ max". $^{2}$ 
Table 3. Individual time-points (TP) of measures of oxidative stress and relevant findings for each study.

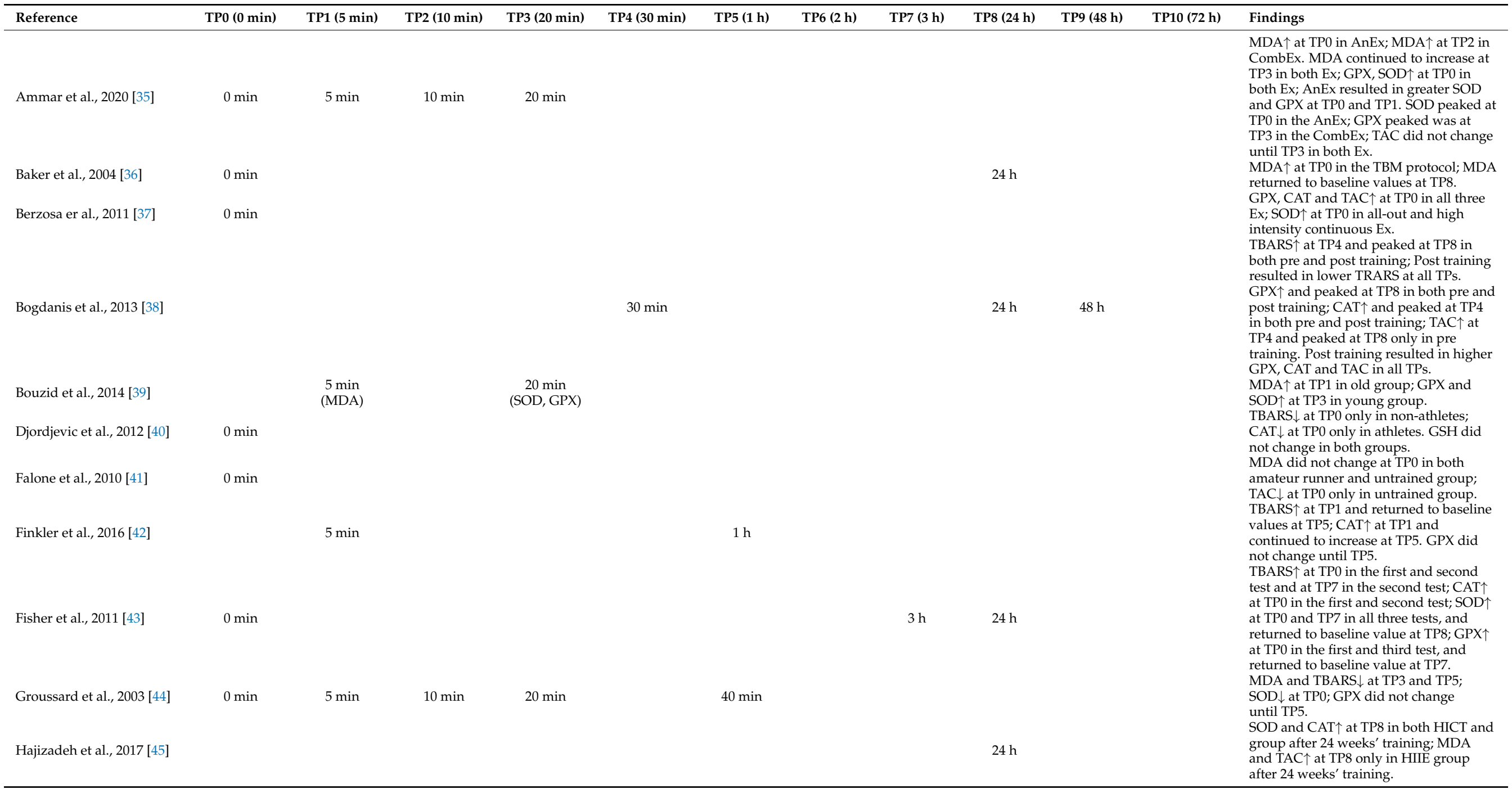


Table 3. Cont.

\begin{tabular}{|c|c|c|c|c|c|c|c|c|c|c|c|c|}
\hline Reference & TP0 (0 min) & TP1 (5 min) & TP2 (10 min) & TP3 (20 min) & TP4 (30 min) & TP5 (1 h) & TP6 (2 h) & TP7 (3 h) & TP8 (24 h) & TP9 (48 h) & TP10 (72 h) & Findings \\
\hline Jammes et al., 2004 [46] & $0 \min$ & $5 \mathrm{~min}$ & $10 \mathrm{~min}$ & $20 \mathrm{~min}$ & $30 \mathrm{~min}$ & & & & & & & $\begin{array}{l}\text { TBARS } \uparrow \text { at TP1 and remained increased } \\
\text { until TP4; GSH } \downarrow \text { at TP1 and returned to } \\
\text { baseline at TP2. }\end{array}$ \\
\hline Jamurtas et al., 2018 [47] & $0 \mathrm{~min}$ & & & & & & & & $24 \mathrm{~h}$ & $48 \mathrm{~h}$ & $72 \mathrm{~h}$ & $\begin{array}{l}\text { TBARS and CAT did not change until } \\
\text { TP10 in both HIIE and HICE; TAC } \uparrow \text { at } \\
\text { TP0 in HICE, and } \uparrow \text { at TP0 and TP8 } \\
\text { in HIIE. }\end{array}$ \\
\hline Kyparos et al., 2007 [48] & $0 \mathrm{~min}$ & & & & & & & & & & & $\begin{array}{l}\text { TBARS, CAT and TAC } \uparrow \text { at TP0; GSH } \downarrow \\
\text { at TP0. } \\
\text { TBARS } \uparrow \text { at TP0 in both pre- and }\end{array}$ \\
\hline Miyazaki et al., 2001 [49] & $0 \mathrm{~min}$ & & & & & & & & & & & $\begin{array}{l}\text { post-training; Post training resulted in } \\
\text { lower TBARS at TPO; SOD, GPX and } \\
\text { CAT did not change at TPO. }\end{array}$ \\
\hline Parker et al., 2014 [50] & $0 \mathrm{~min}$ & & & & & & & & & & & $\begin{array}{l}\text { OS did not change in all three tests; } \\
\text { TAC } \uparrow \text { at TP0 in all three test; } 100 \% \\
\mathrm{VO}_{2} \text { max test resulted in the highest TAC } \\
\text { at TP0. }\end{array}$ \\
\hline Parker et al., 2018 [51] & $0 \mathrm{~min}$ & & & & & $1 \mathrm{~h}$ & $2 \mathrm{~h}$ & $3 \mathrm{~h}$ & & & & $\begin{array}{l}\text { TBARS and SOD } \downarrow \text { at TP0, TP5 and TP6 } \\
\text { in both HIIE and SIE; CAT did not } \\
\text { change until TP7 in both HIIE and SIE. }\end{array}$ \\
\hline $\begin{array}{l}\text { Seifi-Skishahr et al., } \\
2008 \text { [52] }\end{array}$ & $0 \mathrm{~min}$ & & & & & & $2 \mathrm{~h}$ & & $24 \mathrm{~h}$ & & & $\mathrm{MDA} \uparrow$ at TP6. \\
\hline Steinberg et al., 2007 [53] & $0 \mathrm{~min}$ & $5 \mathrm{~min}$ & $10 \mathrm{~min}$ & $20 \mathrm{~min}$ & $30 \mathrm{~min}$ & & & & & & & $\begin{array}{l}\text { TBARS } \uparrow \text { at TP1 and TP2; GSH } \downarrow \text { at TP2 } \\
\text { and TP3. }\end{array}$ \\
\hline Wadley et al., 2016 [54] & $0 \mathrm{~min}$ & & & & $30 \mathrm{~min}$ & & & & & & & $\begin{array}{l}\text { TAC } \uparrow \text { at TP4 relative to TP0 in both } \\
\text { LV-HIIE and HICE. }\end{array}$ \\
\hline
\end{tabular}

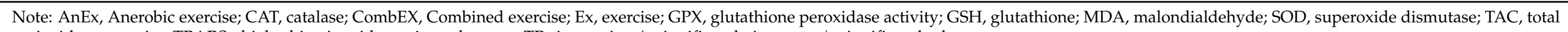
antioxidant capacity; TBARS, thiobarbituric acid reactive substance; TP, time-point; $\uparrow$, significantly increase; $\downarrow$, significantly decrease. 


\subsection{Participants of Selected Studies}

Table 4 outlines the sociodemographic characteristics of participants. Participants mean age in selected studies ranged from 17.3 to 65.1 years old. Participants body mass index ranged from 21.5 to 27.6 . $\mathrm{VO}_{2}$ max of participants ranged from 31.7 to $49.7 \mathrm{~mL} / \mathrm{kg} / \mathrm{min}$. Sixty studies reported no medications and antioxidant dietary supplement prior and throughout the test $[35-38,40-45,47,50-52,54,55]$. Eight studies described participants lifestyle as physically active $[36-38,42,44,45,51,55]$, one study described as physically inactive [35], three studies decried as no regular physical activity [40,43,49], and six studies used sedentary participants $[39,41,46,50,52,53]$. Two studies included groups of trained participants [40,41]; one used handball athletes with regular training [40] and the other used amateur runners [41]. Nine studies used non-smoking participants [40,42,47,50-55], and two studies reported participants refrained from tobacco in the last 6 months $[44,45]$. Finally, eight studies reported participants with no alcohol at least $24 \mathrm{~h}$ prior to the test $[40,41,44,45,47,50,51,54]$.

\subsection{Oxidative Stress Markers}

Seven oxidative stress markers were analyzed, these included: thiobarbituric acid reactive substances (TBARS) $(9 / 21)$, malondialdehyde (MDA) $(8 / 21)$, glutathione (GSH) $(4 / 21)$, glutathione peroxidase (GPX) $(8 / 21)$, superoxide dismutase (SOD) $(8 / 21)$, catalase (CAT) (10/21), and total antioxidant capacity (TAC) $(8 / 21)$ (Table 5).

\subsection{Exercise Modes}

In terms of exercise modality, cycling on ergometers were the most common HIE used, three used treadmills $[39,41,52]$, and one used a shuttle run [48]. In terms of exercise intensity, there were nine studies that included incremental exercise [37,39-42,46,49,50,53], six performed a single bout of exhaustive/maximal exercises [35-37,44,48,55], six implemented interval exercises [38,43,45,47,51,54], and five conducted continuous exercise $[35,37,47,52,54]$. The duration of a single high intensity exercise bout ranged from $20 \mathrm{~s}$ to $5 \mathrm{~min}$.

\subsection{Levels of Evidence}

Conclusive strong evidence was obtained in the selected high-quality samples. In terms of acute oxidative stress assessed immediately following a bout of HIE, among these studies, oxidative stress was significantly increased in five high-quality studies $[35,36,39,48,49]$, and antioxidant status was significantly stimulated in seven high quality studies $[35,37,39,41,47,48,51]$. Moderate evidence on significantly decreased oxidative damage was found in two high quality studies [40,51]. However, the evidence that antioxidant status was not affected immediately after HIE is also strong due to three high-quality studies $[40,49,54]$.

In relation to the effects of different protocols and measurement time on oxidative stress, we observed the following results: 
Table 4. Sociodemographic characteristics of participants.

\begin{tabular}{|c|c|c|c|c|c|c|c|c|c|c|}
\hline Reference & $\begin{array}{l}\text { Age } \\
\text { (Years Old) }\end{array}$ & Gender & $\begin{array}{l}\text { Weight } \\
\text { (kg) }\end{array}$ & BMI & $\begin{array}{l}\mathrm{VO}_{2} \max \\
(\mathrm{mL} / \mathrm{kg} / \mathrm{min})\end{array}$ & Diet & Lifestyle & $\begin{array}{l}\text { Socio-Economic } \\
\text { Level }\end{array}$ & Tobacco & Alcohol \\
\hline Ammar et al., 2020 [35] & $19.5 \pm 1.7$ & male & $71.8 \pm 2.1$ & - & - & $\begin{array}{l}\text { no medications } \\
\text { and antioxidant } \\
\text { dietary } \\
\text { supplement } \\
\text { no medications }\end{array}$ & $\begin{array}{l}\text { physically } \\
\text { inactive }\end{array}$ & - & - & - \\
\hline Baker et al., 2004 [36] & $23 \pm 2$ & male & $75.3 \pm 11$ & - & - & $\begin{array}{l}\text { and antioxidant } \\
\text { dietary } \\
\text { supplement } \\
\text { no medications }\end{array}$ & physically active & $\begin{array}{l}\text { university } \\
\text { student }\end{array}$ & - & - \\
\hline Berzosa et al., 2011 [37] & $23 \pm 0.41$ & male & $75.25 \pm 2.84$ & $23.72 \pm 0.69$ & $43.8 \pm 1.58$ & $\begin{array}{l}\text { and antioxidant } \\
\text { dietary } \\
\text { supplement } \\
\text { no medications }\end{array}$ & physically active & - & - & - \\
\hline Bogdanis et al., 2013 [38] & $24.3 \pm 1.4$ & male & $77.9 \pm 2.9$ & - & - & $\begin{array}{l}\text { and antioxidant } \\
\text { dietary } \\
\text { supplement }\end{array}$ & physically active & - & - & - \\
\hline Bouzid et al., 2014 (young) [39] & $20.3 \pm 2.8$ & $\begin{array}{l}9 \text { males / } \\
6 \text { females }\end{array}$ & $66.1 \pm 11.7$ & - & $44.2 \pm 5.2$ & - & sedentary & - & - & - \\
\hline Bouzid et al., 2014 (old) [39] & $65.1 \pm 3.57$ & $\begin{array}{l}7 \text { males/ } \\
8 \text { females }\end{array}$ & $71.8 \pm 7.6$ & $23.2 \pm 4.4$ & - & - & sedentary & - & - & - \\
\hline Djordjevic et al., 2012 (athletes) [40] & $17.3 \pm 0.2$ & male & $80.9 \pm 1.4$ & $23.9 \pm 0.3$ & $44.6 \pm 0.9$ & $\begin{array}{l}\text { no medications } \\
\text { and antioxidant } \\
\text { dietary } \\
\text { supplement } \\
\text { no medications }\end{array}$ & regular training & - & non-smoking & $\begin{array}{l}\text { no alcohol } 48 \mathrm{~h} \\
\text { before test }\end{array}$ \\
\hline Djordjevic et al., 2012 (non-athletes) [40] & $17.3 \pm 0.3$ & male & $81.6 \pm 6.1$ & $23.6 \pm 1.3$ & $39.7 \pm 1.3$ & $\begin{array}{l}\text { and antioxidant } \\
\text { dietary } \\
\text { supplement }\end{array}$ & $\begin{array}{l}\text { no regular } \\
\text { physical activity }\end{array}$ & - & non-smoking & $\begin{array}{l}\text { no alcohol } 48 \mathrm{~h} \\
\text { before test }\end{array}$ \\
\hline Falone et al., 2010 (amateur runner) [41] & $42 \pm 1$ & male & - & $23.5 \pm 0.5$ & $48.5 \pm 0.9$ & $\begin{array}{l}\text { no medications } \\
\text { and antioxidant } \\
\text { dietary } \\
\text { supplement } \\
\text { no medications }\end{array}$ & regular training & - & - & no alcohol \\
\hline Falone et al., 2010 (untrained) [41] & $39 \pm 3$ & male & - & $26.1 \pm 1.1$ & $33.3 \pm 1.2$ & $\begin{array}{l}\text { and antioxidant } \\
\text { dietary } \\
\text { supplement }\end{array}$ & sedentary & no manual labor & - & no alcohol \\
\hline Finkler et al., 2016 [42] & 26.8 & male & 77.9 & 23.4 & 48.9 & $\begin{array}{l}\text { no medications } \\
\text { and antioxidant } \\
\text { dietary } \\
\text { supplement }\end{array}$ & physically active & - & non-smoking & - \\
\hline
\end{tabular}


Table 4. Cont.

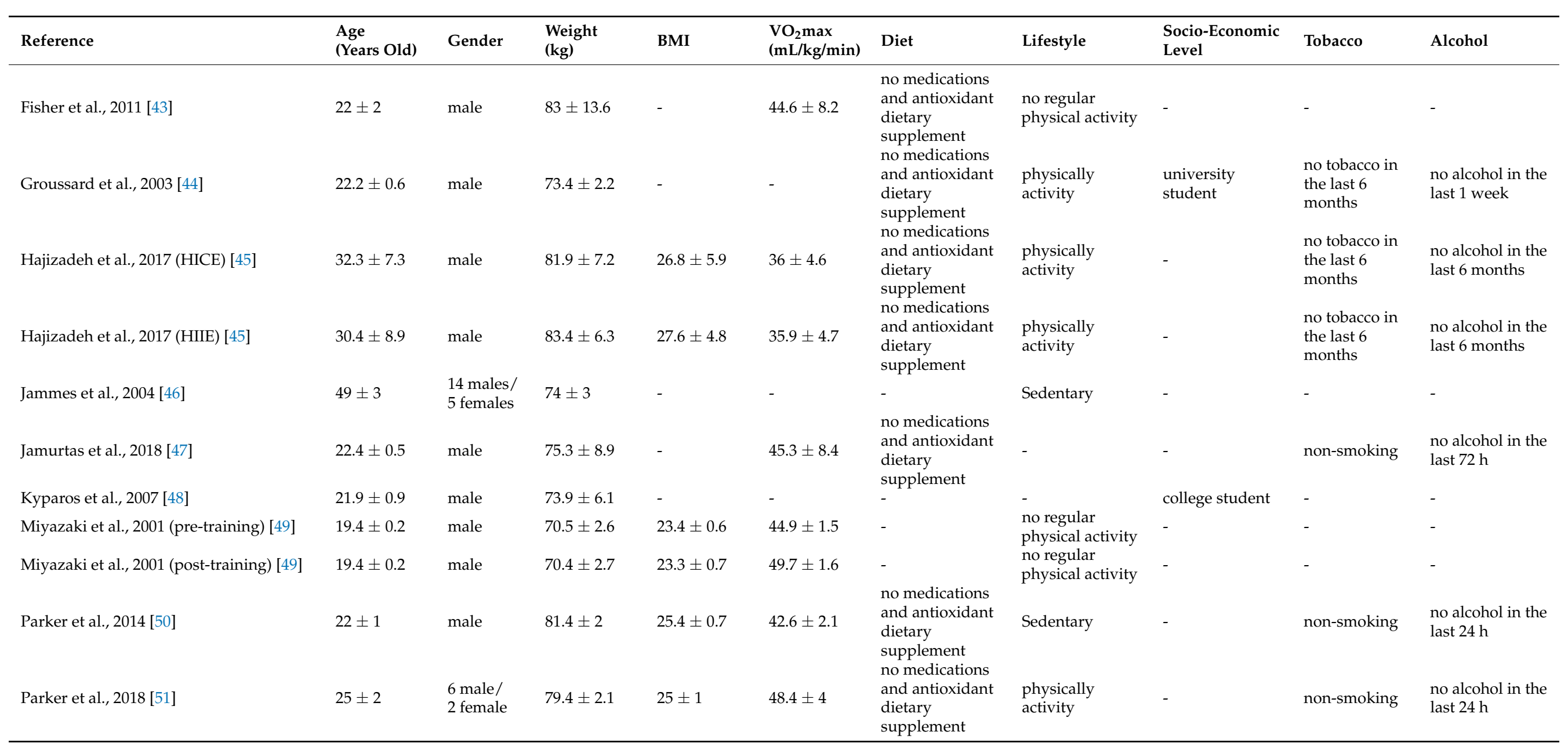


Table 4. Cont.

\begin{tabular}{|c|c|c|c|c|c|c|c|c|c|c|}
\hline Reference & $\begin{array}{l}\text { Age } \\
\text { (Years Old) }\end{array}$ & Gender & $\begin{array}{l}\text { Weight } \\
\text { (kg) }\end{array}$ & BMI & $\begin{array}{l}\mathrm{VO}_{2} \max \\
(\mathrm{mL} / \mathrm{kg} / \mathrm{min})\end{array}$ & Diet & Lifestyle & $\begin{array}{l}\text { Socio-Economic } \\
\text { Level }\end{array}$ & Tobacco & Alcohol \\
\hline Seifi-Skishahr et al., 2008 [52] & $24.1 \pm 3.1$ & - & $71.9 \pm 9.8$ & - & $34.1 \pm 2.7$ & $\begin{array}{l}\text { no medications } \\
\text { and antioxidant } \\
\text { dietary } \\
\text { supplement }\end{array}$ & Sedentary & - & non-smoking & - \\
\hline Steinberg et al., 2007 [53] & $42 \pm 4$ & $\begin{array}{l}9 \text { males / } \\
6 \text { females }\end{array}$ & $70 \pm 3$ & $22 \pm 2$ & $31.7 \pm 2.5$ & - & Sedentary & - & non-smoking & - \\
\hline Wadley et al., 2016 [54] & $22 \pm 3$ & male & - & $24 \pm 3.1$ & $42.7 \pm 5$ & $\begin{array}{l}\text { no medications } \\
\text { and antioxidant } \\
\text { dietary } \\
\text { supplement } \\
\text { no medications }\end{array}$ & - & - & non-smoking & $\begin{array}{l}\text { no alcohol in the } \\
\text { last } 48 \mathrm{~h}\end{array}$ \\
\hline Wiecek et al., 2018 (female) [55] & $22 \pm 0.5$ & female & $59.8 \pm 2.1$ & $21.5 \pm 0.6$ & - & $\begin{array}{l}\text { and antioxidant } \\
\text { dietary } \\
\text { supplement }\end{array}$ & $\begin{array}{l}\text { physically } \\
\text { activity }\end{array}$ & - & non-smoking & - \\
\hline Wiecek et al., 2018 (male) [55] & $21.6 \pm 0.4$ & male & $77.1 \pm 2.7$ & $23.7 \pm 0.5$ & - & $\begin{array}{l}\text { no medications } \\
\text { and antioxidant } \\
\text { dietary } \\
\text { supplement }\end{array}$ & $\begin{array}{l}\text { physically } \\
\text { activity }\end{array}$ & - & non-smoking & - \\
\hline
\end{tabular}


Table 5. Oxidative stress markers.

\begin{tabular}{|c|c|c|c|c|c|c|c|c|c|}
\hline \multirow{2}{*}{ Reference } & \multirow{2}{*}{ Sample Size } & \multicolumn{8}{|c|}{ Acute Response on Oxidative Stress and Antioxidant Status } \\
\hline & & MDA & TBARS & OS & TAC & CAT & SOD & GPX & GSH \\
\hline Ammar et al., 2020 [35] (anerobic) & 10 & $\operatorname{sig} \uparrow$ & - & - & $\mathrm{ns} \uparrow$ & - & $\operatorname{sig} \uparrow *$ & $\operatorname{sig} \uparrow *$ & - \\
\hline Ammar et al., 2020 [35] (combined) & 10 & ns $\uparrow$ & - & - & ns $\uparrow$ & - & $\operatorname{sig} \uparrow *$ & $\operatorname{sig} \uparrow *$ & - \\
\hline Baker et al., 2004 [36] (TBM) & 18 & $\operatorname{sig} \uparrow *$ & - & - & - & - & - & - & - \\
\hline Baker et al., 2004 [36] (FFM) & 18 & ns $\uparrow *$ & - & - & - & - & - & - & - \\
\hline Berzosa er al., 2011 [37] (incremental) & 34 & - & - & - & $\operatorname{sig} \uparrow$ & $\operatorname{sig} \uparrow$ & $\mathrm{ns} \uparrow$ & $\operatorname{sig} \uparrow$ & - \\
\hline Berzosa er al., 2011 [37] (all-out) & 34 & - & - & - & $\operatorname{sig} \uparrow$ & $\operatorname{sig} \uparrow$ & $\operatorname{sig} \uparrow$ & $\operatorname{sig} \uparrow$ & - \\
\hline Berzosa er al., 2011 [37] $\left(70 \% \mathrm{VO}_{2} \max \right)$ & 34 & - & - & - & $\operatorname{sig} \uparrow$ & $\operatorname{sig} \uparrow$ & $\operatorname{sig} \uparrow$ & $\operatorname{sig} \uparrow$ & - \\
\hline Bogdanis et al., 2013 [38] (pre-training) & 8 & - & $\operatorname{sig} \uparrow$ & - & $\operatorname{sig} \uparrow$ & $\operatorname{sig} \uparrow$ & - & ns $\uparrow$ & - \\
\hline Bogdanis et al., 2013 [38] (post-training) & 8 & - & $\operatorname{sig} \uparrow$ & - & $\mathrm{ns} \uparrow$ & $\operatorname{sig} \uparrow$ & - & ns $\uparrow$ & - \\
\hline Bouzid et al., 2014 [39] (young) & 15 & $\mathrm{~ns} \uparrow *$ & - & - & - & - & $\operatorname{sig} \uparrow *$ & $\operatorname{sig} \uparrow$ & - \\
\hline Bouzid et al., 2014 [39] (old) & 15 & $\operatorname{sig} \uparrow *$ & - & - & - & - & ns $\uparrow *$ & ns $\uparrow$ & - \\
\hline Djordjevic et al., 2012 [40] (athletes) & 58 & - & $\mathrm{ns} \uparrow$ & - & - & $\operatorname{sig} \downarrow$ & ns $\downarrow$ & - & $\mathrm{ns} \uparrow$ \\
\hline Djordjevic et al., 2012 [40] (non-athletes) & 37 & - & $\operatorname{sig} \downarrow$ & - & - & ns & ns $\uparrow$ & - & ns $\uparrow$ \\
\hline Falone et al., 2010 [41] (amateur runner) & 33 & $\mathrm{~ns} \downarrow *$ & - & - & $\mathrm{ns}$ * & - & - & - & - \\
\hline Falone et al., 2010 [41] (untrained) & 25 & ns $\uparrow *$ & - & - & $\operatorname{sig} \downarrow *$ & - & - & - & - \\
\hline Finkler et al., 2016 [42] & 32 & - & $\operatorname{sig} \uparrow$ & - & - & $\operatorname{sig} \uparrow$ & - & $\mathrm{ns} \uparrow$ & - \\
\hline Fisher et al., 2011 [43] (first) & 8 & $\operatorname{sig} \uparrow$ & $\operatorname{sig} \uparrow$ & - & - & $\operatorname{sig} \uparrow$ & $\operatorname{sig} \uparrow$ & $\operatorname{sig} \uparrow$ & - \\
\hline Fisher et al., 2011 [43] (second) & 8 & $\operatorname{sig} \uparrow$ & $\operatorname{sig} \uparrow$ & - & - & $\operatorname{sig} \uparrow$ & $\operatorname{sig} \uparrow$ & ns $\uparrow$ & - \\
\hline Fisher et al., 2011 [43] (third) & 8 & $\mathrm{~ns} \uparrow$ & $\mathrm{ns} \uparrow$ & - & - & ns $\uparrow$ & $\operatorname{sig} \uparrow$ & $\operatorname{sig} \uparrow$ & - \\
\hline Groussard et al., 2003 [44] & 8 & ns $\downarrow$ & $\mathrm{ns} \downarrow$ & - & - & - & $\operatorname{sig} \downarrow$ & ns & - \\
\hline Hajizadeh et al., 2017 [45] (HICE) & 62 & - & - & - & - & - & - & - & - \\
\hline Hajizadeh et al., 2017 [45] (HIIE) & 65 & - & - & - & - & - & - & - & - \\
\hline Jammes et al., 2004 [46] & 19 & - & $\mathrm{ns} \uparrow$ & - & - & - & - & - & ns $\downarrow$ \\
\hline Jamurtas et al., 2018 [47] (HIIE) & 12 & - & $\mathrm{ns} \uparrow$ & - & $\operatorname{sig} \uparrow *$ & $\mathrm{~ns} \uparrow$ & - & - & - \\
\hline Jamurtas et al., 2018 [47] (HICE) & 12 & - & $\mathrm{ns} \uparrow$ & - & $\operatorname{sig} \uparrow *$ & $\mathrm{~ns} \uparrow$ & - & - & - \\
\hline Kyparos et al., 2007 [48] & 11 & - & $\operatorname{sig} \uparrow$ & - & $\operatorname{sig} \uparrow$ & $\operatorname{sig} \uparrow$ & - & - & $\operatorname{sig} \downarrow$ \\
\hline Miyazaki et al., 2001 [49] (pre-training) & 9 & - & $\operatorname{sig} \uparrow *$ & - & - & ns & $\mathrm{ns} \uparrow$ & $\mathrm{ns} \uparrow$ & - \\
\hline Miyazaki et al., 2001 [49] (post-training) & 9 & - & $\operatorname{sig} \uparrow *$ & - & - & ns & $\mathrm{ns} \uparrow$ & ns $\downarrow$ & - \\
\hline Parker et al., 2014 [50] (70\% $\left.\mathrm{VO}_{2} \max \right)$ & 14 & - & - & $\mathrm{ns} \uparrow$ & $\operatorname{sig} \uparrow *$ & - & - & - & - \\
\hline Parker et al., 2014 [50] (85\% $\left.\mathrm{VO}_{2} \max \right)$ & 14 & - & - & $\mathrm{ns} \uparrow$ & $\operatorname{sig} \uparrow$ & - & - & - & - \\
\hline Parker et al., 2014 [50] (100\% $\left.\mathrm{VO}_{2} \max \right)$ & 14 & - & - & $\mathrm{ns} \uparrow$ & $\operatorname{sig} \uparrow *$ & - & - & - & - \\
\hline Parker et al., 2018 [51] (HIIE) & 8 & - & $\operatorname{sig} \downarrow$ & - & - & $\mathrm{ns} \uparrow$ & $\operatorname{sig} \downarrow$ & - & - \\
\hline Parker et al., 2018 [51] (SIE) & 8 & - & $\operatorname{sig} \downarrow$ & - & - & $\mathrm{ns} \uparrow$ & $\operatorname{sig} \downarrow$ & - & - \\
\hline Seifi-Skishahr et al., 2008 [52] & 10 & $\mathrm{~ns} \uparrow$ & - & - & - & - & - & - & - \\
\hline Steinberg et al., 2007 [53] & 15 & ns $\uparrow$ & - & - & - & - & - & - & ns $\downarrow$ \\
\hline Wadley et al., 2016 [54] (LV-HIIE) & 10 & - & - & - & ns $\downarrow$ & - & - & - & - \\
\hline Wadley et al., 2016 [54] (HICE) & 10 & - & - & - & ns $\downarrow$ & - & - & - & - \\
\hline Wiecek et al., 2018 [55] & 20 & - & - & - & - & $\mathrm{ns} \uparrow$ & ns $\uparrow$ & ns $\downarrow$ & - \\
\hline
\end{tabular}

Note: CAT, catalase; FFM, fat-free mass; GPX, glutathione peroxidase activity; GSH, glutathione; HICE, high-intensity continuous exercise; HIIE, high-intensity interval exercise; LV-HIIE, low-volume high-intensity interval exercise; MDA, malondialdehyde; ns, not significant; SIE, sprint interval exercise; sig, significant; SOD, superoxide dismutase; TAC, total antioxidant capacity; TBARS, thiobarbituric acid reactive substance; $\mathrm{TBM}$, total body mass; $\mathrm{VO}_{2} \mathrm{max}$, maximal oxygen uptake; ${ }^{*}$, significant difference between groups.

\subsubsection{Acute Effect of Oxidative Stress and Antioxidant Status after HIE}

Seventeen studies assessed the acute response of oxidative stress immediately following HIE (TP0 and TP1). Among them, nine studies reported significantly increased acute oxidative damage immediately post HIE [35,36,38,39,42-44,48,49]. Four studies reported significant acute oxidative damage following a maximal exercise [35,36,44,48]. Three studies used incremental protocols [39,42,49] and another two used intermittent protocols $[38,43]$. On the contrary, only two studies reported significantly decreased acute oxidative damage [40,51]. One study used incremental exercise [40], and the other study adopted an interval protocol. Six studies did not observe significant changes $[41,46,47,50,52,53]$. Three of them used incremental protocols [41,46,53], one of them executed high intensity interval exercise [50], and one of them used high-intensity continuous exercise [52]. The remaining one compared interval exercise with continuous exercise performed at a high intensity, indicating no between-group differences regarding acute oxidative responses [47]. It was worth noting that one study also used electron spin resonance (ESR) to directly test the production of lipid free radicals to study oxidative stress responses to maximal 
exercise [44]. The authors found that lipid free radicals increased significantly after exercise, while plasma TBARS concentrations did not increase.

For antioxidant status immediately after HIE, data were available from eighteen studies. Twelve studies reported the alterations of redox homeostasis. Most studies $(9 / 18)$ indicated elevated antioxidant enzyme activities $[35,37-39,42,43,47,48,50]$. There were four studies that used interval exercise $[38,43,47,50]$. Three studies used incremental protocols $[37,39,42]$ and three studies used maximal exercises $[35,37,48]$. Two performed high-intensity continuous exercises [37,47] and only one applied a combined protocol, consisting of maximal exercise followed by a moderate continuous exercise [35]. Among them, four studies investigated antioxidative responses between different types of protocols $[35,37,47,50]$. In contrast, three studies indicated decreased antioxidant activities [41,44,51]. Incremental [41], interval [51], and maximal [44] protocols were used. Six studies reported no changes in antioxidant activities immediately after HIE [40,46,49,53-55]. Four of them performed incremental protocols $[40,46,49,53]$. One used maximal exercise [55] and the remaining study compared antioxidant changes between high-intensity interval and high-intensity continuous exercises [54].

\subsubsection{Time of Measurement Effects of Oxidative Stress and Antioxidant Status after HIE}

Eleven studies used multiple post-exercise measures on oxidative markers after exercise. Eight studies observed increased oxidative damage following HIE [35,36,38,42,43,46,52,53]. Among them, increased oxidative damage was observed from TP0 (0 min) to TP9 (48 h) following HIE, with peak values at various time points. Peak oxidative damage occurred at TP1 (5 $\mathrm{min})$ in two studies [46,53]. One study reported peak values at TP6 (2 h) [52]. Another study observed the highest oxidative damage at TP8 (24 h) [38]. No conclusive peak value could be found in four studies $[35,36,42,43]$. Generally, oxidative damage returned to baseline within $24 \mathrm{~h}$ after HIE in most studies $(5 / 8)[36,42,43,52,53]$. Only one study reported increased damage after $24 \mathrm{~h}$ [38]. No recovery data could be recorded in the other two studies due to the limited measurement time $[35,46]$.

Two studies reported decreased oxidative stress markers from TP0 (0 min) to TP6 (2 h) $[44,51]$. Only one study indicated no change in oxidative stress at any measurement time from TP0 (0 $\mathrm{min})$ to TP10 $(72 \mathrm{~h})$ [47].

For endogenous redox status, data was analyzed from eleven studies [35,38,42-44,46,47,51,53-55]. All studies reported significant changes in antioxidants from TP0 (0 min) to TP4 (30 min). Seven studies indicated early alterations within 5 min after HIE [35,42-44,46,47,51], while two studies did not observe significant changes until $30 \mathrm{~min}[38,54]$. Another two studies indicated altered antioxidant status from $10 \mathrm{~min}$ to $15 \mathrm{~min}$ following HIE $[51,53]$.

\section{Discussion}

This systematic review aimed to investigate the effects of HIE on oxidative stress and antioxidant capacity in untrained adults. The results suggest that HIE induces oxidative stress compared to a resting state. Regardless of whether HIE is performed on treadmills, cycle ergometers, or other exercise types, if the duration is more than $30 \mathrm{~s}$ and $\mathrm{VO}_{2}$ max reaches $70 \%$ or more, the balance of oxidative and antioxidant systems in the body will be disrupted, leading to oxidative stress and cellular damage. The results also show that regular exercise, sufficient recovery, and young age increased protection against exerciseinduced oxidative damage; however, further studies are needed to confirm and explore this finding.

HIE can interfere with the balance between oxidation and anti-oxidation systems in the body. During HIE or during a short period following HIE, the production of ROS significantly increases, which is related to the sharp increase in oxygen consumption, activation of inflammatory cells, and contraction of muscle. However, the endogenous antioxidant capacity was simultaneously elevated. 
HIE can be either aerobic or anaerobic exercise, or a combination of both. Incremental exercise, maximal exercise, intermittent exercise and high intensity continuous exercise at $70 \% \mathrm{VO}_{2}$ max or above were the common HIE methodologies used in untrained adults.

Studies that refer to different types of HIE report the almost conclusive finding that (1) HIE induces oxidative stress, (2) HIE-induced oxidative stress is transient, (3) antioxidant capacity is also activated after HIE, (4) regular exercise enhanced the antioxidant defense mechanisms, (5) HIE-induced oxidative stress is related to individual subject characteristics.

\subsection{HIE Induces Oxidative Stress}

HIE induces oxidative stress, regardless of the mode of exercise. In this review, cycling and running were the more common exercises used. A cycling exercise to exhaustion can induce oxidative stress [35-38,42,43,46,47,49,50,53-55] and a running exercise to exhaustion has a similar effect $[39,41,48,52]$. This has been evidenced by a recent study [56], in which oxidative stress was assessed using two different HIE modalities: running and cycling. The study concluded that both cycling and running induce oxidative stress, even though TAC recovers faster among runners.

Furthermore, the intensity of HIE can also influence oxidative stress. Using an incremental intensity, Parker et al. (2014) observed significant oxidative stress at intensities of $70 \% \mathrm{VO}_{2}$ max or above with increasing oxidative stress accompanied by increased exercise intensity [50]. This has also been reported by Fogarty et al. (2011), who conducted three aerobic exercises at $40 \%, 70 \%$, and $100 \%$ of $\mathrm{VO}_{2} \max$ [57]. Oxidative damage to DNA was increased at $70 \%$ and $100 \% \mathrm{VO}_{2}$ max and the extent of damage was positively related to the intensity. Similarly, during a 30 s maximal cycling test, the selection of resistive forces (TBM or FFM) may induce different metabolic responses for oxidative stress [36]. This study indicated that a FFM protocol was metabolically more efficient compared to the TBM protocol and produced less oxidative stress and muscle damage during exercise. It can be concluded that when the exercise intensity is higher than $70 \% \mathrm{VO}_{2}$ max, significant oxidative stress occurs, and the extent of oxidative damage is positively related to the intensity. This finding is consistent with the recent work of Tryfidou et al. (2020), who reported DNA oxidative stress damage increased after exercise with intensities higher than $75 \%$ [58].

The duration of HIE can also be one of the most important factors for exercise-induced oxidative stress. Using data from this review, we observed that for a single high-intensity exercise bout, oxidative stress occurs when the exercise duration is more than $30 \mathrm{~s}$. The most common HIE protocol consists of several $30 \mathrm{~s}$ "all-out" bouts separated by recovery. As such, we believe that most HIE protocols will induce oxidative stress. However, a single bout duration shorter than $30 \mathrm{~s}$ was proven to be associated with less oxidative stress [59]. With the same modality and intensity. Cipryan (2017) performed 3 HIE protocols with a total of 12 min exercise [59]. The durations were $15 \mathrm{~s}, 30 \mathrm{~s}$, or $60 \mathrm{~s}$, respectively, and the work/rest ratio was 1 . The authors observed an immediate increase in oxidative stress markers in all three protocols. Among them, oxidative stress in 30/30 protocol showed the smallest increases, while the TAC in 15/15 protocol demonstrated the largest increase. However, further studies are needed to investigate oxidative damage following exercises performed at the same intensity but using different modalities (continuous or intermittent).

\subsection{HIE-Induced Oxidative Stress Is Transient}

Using data from this review, we conclude that there is a significant increase in oxidative stress markers following exercise at TP0 $(0 \mathrm{~h})$ when compared to rest. To investigate oxidative damage after exercise at multiple TP, data were available from 13 studies (details in Table 3). This review demonstrates the acute effect on oxidative stress following highintensity exercise often occurs within $5 \mathrm{~min}$ at the end of the exercise, remaining elevated within 30 min following exercise. Moreover, several studies reported that oxidative stress peaked at 5 min post exercise. Most of the increased oxidative damage returned to basal level within $24 \mathrm{~h}$ following exercise cessation [36,42,43,52,53]. Researchers observed that 
the greatest oxidative stress occurred in healthy subjects 5 min post exercise and then recovered gradually, with different markers recovering at different rates [46,53].

Basically, responses of the antioxidant enzymes to oxidative stress are lagging and lasting. Significant changes of SOD, CAT, and TAC were observed at $15 \mathrm{~min}$ after exercise [55]. SOD was increased continuously until $3 \mathrm{~h}$ of the end of exercise [43]. Finkler et al. (2016) also indicated that TBARS concentrations increased immediately, whereas the activation of CAT appears to be small and continues to increase during the recovery period [42]. Similarly, TAC is significantly elevated from rest to post exercise and remained above pre-exercise levels for $24 \mathrm{~h}$ [59-61]. This agrees with the previous study. Farney et al. (2012) reported an absence of oxidative stress in trained men following HIE [62]. One study reported significant changes in antioxidant activities were found $20 \mathrm{~min}$ post exercise [63]. Therefore, stimulated antioxidant defense could be observed or not at the cessation of HIE.

\subsection{The Antioxidant Capacity Is also Activated after HIE}

The degree of oxidative stress depends on the balance between the generation of ROS and the effectiveness of the antioxidant defense system. Studies have shown that the antioxidant defense system in the body was rapidly activated after HIE [37,44,47,48,50,64,65]. Fisher et al. (2011) also found that the absence of lymphocyte cell viability decreases after HIE exercise and was due to the increased activity of antioxidant enzymes in lymphocytes [43]. Many studies have also shown a significant increase in antioxidant activity after HIE compared to baseline values [38,43,46,59-61,66-68]. In contrast, several studies found that antioxidant enzyme activities did not increase but decreased after HIE. Others, Falone et al. (2010) and Kröpfl et al. (2021), also found that some endogenous antioxidants did not change after exercise $[41,56]$. This supported one of our observations that antioxidative status did not change after HIE. Such results demonstrate that oxidative stress caused by HIE had been rapidly neutralized by the antioxidant system, and that there was no significant change in antioxidant markers.

Whether moderate oxidative stress, which stimulates the antioxidative system temporarily and appropriately, contributes to the improvement of antioxidant levels in the body deserves further investigation.

\subsection{Regular Exercise Enhanced the Antioxidant Defense Mechanisms}

Individuals who are physically active have better antioxidant systems and can respond to oxidative stress induced by HIE more quickly than sedentary individuals. In the resting state, physically active people have higher baseline values of TAC, and lower TBARS $[40,41,60,65]$. After exercise, TAC showed the largest changes, indicating that regular exercise can improve the activity of the antioxidant system and reduce exercise-induced oxidative damage. It also shows that under the same HIE protocol, the higher the fitness levels of individuals, the less obvious the oxidative stress increases should be after exercise. In addition, physically active individuals have stronger ability to counter oxidative stress, affecting the recovery time of oxidative indicators, such as TBARS, MDA, and TAC, which did not change immediately after exercise [22,64,69-71]. Even in the study of Groussard et al. (2003), a sharp post-exercise decrease was observed in plasma TBARS and MDA levels in university physical education students [44]. This is more likely to happen in individuals who are physically active.

Compared with a single bout of HIE, however, short-term HIE leads to different conclusions in relation to the effects on oxidative stress. Faruk et al. (2013) conducted a 10-day HIE protocol and concluded that HIE could improve the oxidative stress of participants [72]. On the contrary, a HIE protocol lasting 3 weeks with training frequency of 3 times weekly and the total training time over $2 \mathrm{~h}$ was proven to reduce oxidative stress and to upregulate the antioxidant system [38]. In the study of Fisher et al. (2011), three HIE protocols were completed with 2 days' recovery between each session [43]. The authors found that oxidative stress occurred on the first and second session but did not significantly increase in the third session. Similar findings were reported by Miyazaki 
et al. (2001) [49]. Furthermore, Falone et al. (2010) specified that long-term regular and moderate aerobic physical activity can increase antioxidant capacity [41]. Similar findings were provided by Hajizadeh et al. (2017) [45]. After a long period of moderate intensity continuous training, high-intensity continuous training and high-intensity interval training, the authors indicated three types of interventions attenuated oxidative stress with different kinetics and moderate intensity continuous training was superior in the promotion of antioxidant capacity.

Using the evidence from the studies reviewed, we can infer that a single session of HIE can induce oxidative stress; however, a HIE protocol lasting a longer period will attenuate oxidative stress. This statement agrees with the findings that regular HIE could keep oxidative DNA damage at a lower level for a long period [73] and consecutive days of high-intensity exercise improved endogenous antioxidant capacity and reduced exerciseinduced oxidative stress [74]. However, further studies are needed to explore the efficiency of long-term high intensity exercise and moderate intensity exercise on antioxidant capacity.

\subsection{HIE-Induced Oxidative Stress Is Related to Individual Characteristics}

In terms of gender, oxidative stress in both male and female individuals changed following HIE [72]. In the study of Jammes et al. (2004), the maximum increase in plasma TBARS after exercise was slightly higher in men than in women, while this difference was almost negligible [46]. Furthermore, Wiecek et al. (2018) indicated no differences concerning changes in antioxidant activity post HIE between males and females, while males represented higher level of baseline antioxidant activity [55]. However, Steinberg et al. (2007) and Jammes et al. (2004) observed that maximal increase in TBARS was positively related to $\mathrm{VO}_{2} \max [46,53]$. From these studies, we can conclude that high-intensity exerciseinduced oxidative stress is not related to gender. Since limited studies focused on women, more studies exploring oxidative stress using different gender participants are needed.

Generally, aging is related to a decline in antioxidant capacity and aged populations are more susceptible to oxidative stress. Bouzid et al. (2014) compared changes in oxidative stress with aging populations at rest and post HIE [39]. There was no difference in oxidative markers between young and elderly groups at rest and antioxidant activities only increased in the young group post exercise. Furthermore, Boisseau et al. (2000) found that post-pubertal boys have greater muscle mass, higher mitochondrial respiration, and greater oxygen uptake during exercise [75]. This resulted in greater ROS production and subsequent oxidative stress because of puberty. At the same time, post-pubertal boys had higher antioxidant capacity. Higher baseline value of TBARS, CAT, and SOD were observed in post-pubertal populations, and more significant changes in these markers after exercise were found. Therefore, post-pubertal populations were considered to have a stronger ability to counter oxidative stress [67].

The findings from cross-sectional studies are limited. However, longitudinal studies are needed to demonstrate the response of aging and fitness levels in relation to oxidative stress and antioxidant capacity.

\section{Limitations and Strengthens}

There are several limitations in the present review. Firstly, the heterogeneity among selected studies is considerable $\left(\mathrm{I}^{2}>75 \%\right)$. This may be due to the exercise protocols using different characteristics, making it difficult to draw a more precise conclusions regarding exercise type, duration, and intensity. Second, although most studies reported no medications and antioxidant dietary supplement before and during the test, they did not analyze daily diet, which could have an impact on the results. Finally, this review chose to focus solely on studies that have reported oxidative stress assayed from blood concentrations as this represents the most frequently used indicator. We acknowledge that oxidative damage can also be detected directly by ESR or indirectly in urine and muscle indicators. Furthermore, this is the first systematic review investigating the influence of HIE with untrained humans and MQA was executed rigorously through all the selected studies. 


\section{Practical Applications}

- High-intensity exercise-induced oxidative stress is acute and recoverable, and in young healthy untrained humans, oxidative stress after a single bout of high intensity exercise will not be elevated to dangerous levels.

- Higher physical fitness level is associated with shorter time to recovery from the exercises induced oxidative stress.

- Higher intensity is related to higher exercise-induced oxidative stress, and $70 \%$ $\mathrm{VO}_{2}$ max with sufficient recovery is a better exercise mode for untrained humans to initiate high-intensity exercise.

- Establishing a standardized high-intensity exercise protocol in order to specifically investigate oxidative responses post exercise will help provide a better knowledge in this area.

\section{Conclusions}

This systematic review demonstrates that an increase in oxidative damage occurs following a HIE bout. The data further demonstrate that oxidative stress was positively associated with increases in exercise intensity, while benefits were observed in studies using more than one HIE session. Although oxidative stress occurs after HIE, this is not a negative outcome per se. Such exercise-induced oxidative stress is transient and most likely recovers within $24 \mathrm{~h}$, or even sooner, as studies using multiple TP measurements suggest. Such acute oxidative stress does not have any long-term harmful health outcomes. On the contrary, short-term oxidative stress can stimulate the body's antioxidant system, which in the long term will improve the body's antioxidant capacity and have a positive effect on health promotion.

The exercise modality during HIE is not related to oxidative stress, but the intensity and duration of HIE are closely related to increases in oxidative stress. It is generally believed that the greater the intensity and the longer the duration of HIE, the more intense the oxidative stress would be. At the same time, the degree of oxidative stress is also related to the individual's exercise habits, and individual fitness levels and age. Gender also appears to be associated with resting levels of antioxidant capacity, with greater values recorded in males. Individuals who are physically active appear to have greater antioxidant capacities. From the findings of this review, we can conclude that HIE can be an alternative for untrained humans to improve antioxidant capacity and promote health.

However, the combination of frequency, intensity, and duration of HIE protocols needs to consider individual characteristics comprehensively when prescribing individual training programs, since induced oxidative stress level responses are not identical or specific between individuals.

Funding: This research received no external funding.

Institutional Review Board Statement: Not applicable.

Informed Consent Statement: Not applicable.

Data Availability Statement: The data presented in this study are available on request from the corresponding author. The data are not publicly available due to privacy.

Acknowledgments: I would like to show my deepest gratitude to my supervisory team, Huw and Baker, they are respectable, responsible, and resourceful scholars. They have provided me with valuable guidance in every stage of the research. Additionally, I would like to thank Qiaojun Wang from the Faculty of Sport Science, Ningbo University for helping me to complete the selection.

Conflicts of Interest: The authors declare no conflict of interest. 


\section{Appendix A}

Table A1. Methodological quality assessment questions.

\begin{tabular}{ll}
\hline Reporting & Yes $1 /$ No 0 \\
\hline $\begin{array}{l}\text { 1. Is the hypothesis/aim/objective of the study clearly described? } \\
\text { 2. Are the interventions of interest clearly described? Treatments and placebo (where relevant) } \\
\text { that are to be compared should be clearly described. }\end{array}$ & Yes $1 /$ No 0 \\
$\begin{array}{l}\text { 3. Are the characteristics of the subjects included in the study clearly described? } \\
\begin{array}{l}\text { 4. Are the main findings of the study clearly described? Simple outcome data (including } \\
\text { denominators and numerators) should be reported for all major findings so that the reader }\end{array}\end{array}$ & Yes $1 /$ No 0 \\
$\begin{array}{l}\text { can check the major analyses and conclusions. } \\
\text { 5. Have all important adverse events that may be a consequence of the intervention }\end{array}$ & Yes $1 /$ No 0 \\
$\begin{array}{l}\text { Intern reported? } \\
\text { 6. Was an attempt made to blind those measuring the main outcomes of the intervention? }\end{array}$ & Yes $1 /$ No $0 /$ unable to determine 0 \\
7. Were the statistical tests used to assess the main outcomes appropriate? & Yes $1 /$ No $0 /$ unable to determine 0 \\
$\begin{array}{l}\text { Internal validity—confounding (selection bias) } \\
\text { 8. Were study subjects in different intervention groups (trials and cohort studies) or were the } \\
\text { cases and controls (case-control studies) recruited over the same period of time? }\end{array}$ & Yes $1 /$ No $0 /$ unable to determine 0 \\
$\begin{array}{l}\text { 9. Were study subjects randomized to intervention groups? } \\
\text { Power }\end{array}$ & Yes $1 /$ No $0 /$ unable to determine 0 \\
$\begin{array}{l}\text { 10. Did the study have sufficient power to detect an important effect where the probability } \\
\text { value for a difference being due to chance is less than } 5 \% \text { ? }\end{array}$ & Yes $1 /$ No $0 /$ unable to determine 0 \\
Methodological quality assessment questions modified from Downs and Black (1998). &
\end{tabular}

\section{References}

1. Harman, D. Aging: A Theory Based on Free Radical and Radiation Chemistry. J. Gerontol. 1956, 11, 298-300. [CrossRef] [PubMed]

2. Forsberg, L.; de Faire, U.; Morgenstern, R. Oxidative Stress, Human Genetic Variation, and Disease. Arch. Biochem. Biophys. 2001, 389, 84-93. [CrossRef] [PubMed]

3. Gutteridge, J.M.; Mumby, S.; Koizumi, M.; Taniguchi, N. "Free” Iron in Neonatal Plasma Activates Aconitase: Evidence for Biologically Reactive Iron. Biochem. Biophys. Res. Commun. 1996, 229, 806-809. [CrossRef]

4. Halliwell, B. Free radicals, proteins and DNA: Oxidative damage versus redox regulation. Biochem. Soc. Trans. 1996, $24,1023-1027$. [CrossRef]

5. Cooper, C.E.; Vollaard, N.B.; Choueiri, T.; Wilson, M.T. Exercise, free radicals and oxidative stress. Biochem. Soc. Trans. 2002, 30, 280-285. [CrossRef] [PubMed]

6. Banerjee, A.K.; Mandal, A.; Chanda, D.; Chakraborti, S. Oxidant, antioxidant and physical exercise. Mol. Cell. Biochem. 2003, 253, 307-312. [CrossRef]

7. Koska, J.; Blazícek, P.; Marko, M.; Grna, J.D.; Kvetnanský, R.; Vigas, M. Insulin, catecholamines, glucose and antioxidant enzymes in oxidative damage during different loads in healthy humans. Physiol. Res. 2000, 49, 95-100.

8. Cooke, M.S.; Evans, M.D.; Dizdaroglu, M.; Lunec, J. Oxidative DNA damage: Mechanisms, mutation, and disease. FASEB J. 2003, 17, 1195-1214. [CrossRef]

9. Kruk, J.; Aboul-Enein, H.Y.; Kładna, A.; Bowser, J.E. Oxidative stress in biological systems and its relation with pathophysiological functions: The effect of physical activity on cellular redox homeostasis. Free Radic. Res. 2019, 53, 497-521. [CrossRef]

10. Liguori, I.; Russo, G.; Curcio, F.; Bulli, G.; Aran, L.; DELLA-Morte, D.; Gargiulo, G.; Testa, G.; Cacciatore, F.; Bonaduce, D.; et al. Oxidative stress, aging, and diseases. Clin. Interv. Aging 2018, 13, 757-772. [CrossRef]

11. Valko, M.; Rhodes, C.J.; Moncol, J.; Izakovic, M.; Mazur, M. Free radicals, metals and antioxidants in oxidative stress-induced cancer. Chem. Biol. Interact. 2006, 160, 1-40. [CrossRef]

12. Ruzicic, R.D.; Jakovljevic, V.; Djordjevic, D. Oxidative Stress in Training, Overtraining and Detraining: From Experimental to Applied Research. Serbian J. Exp. Clin. Res. 2016, 17, 343-348. [CrossRef]

13. Davies, K.J.; Quintanilha, A.T.; Brooks, G.A.; Packer, L. Free radicals and tissue damage produced by exercise. Biochem. Biophys. Res. Commun. 1982, 107, 1198-1205. [CrossRef]

14. Alessio, H.M. Exercise-induced oxidative stress. Med. Sci. Sports Exerc. 1993, 25, 218-224. [CrossRef]

15. Radak, Z.; Sasvári, M.; Nyakas, C.; Taylor, A.W.; Ohno, H.; Nakamoto, H.; Goto, S. Regular Training Modulates the Accumulation of Reactive Carbonyl Derivatives in Mitochondrial and Cytosolic Fractions of Rat Skeletal Muscle. Arch. Biochem. Biophys. 2000, 383, 114-118. [CrossRef] [PubMed]

16. Radak, Z.; Naito, H.; Kaneko, T.; Tahara, S.; Nakamoto, H.; Takahashi, R.; Cardozo-Pelaez, F.; Goto, S. Exercise training decreases DNA damage and increases DNA repair and resistance against oxidative stress of proteins in aged rat skeletal muscle. Pflügers Arch. 2002, 445, 273-278. [CrossRef] [PubMed] 
17. Radak, Z.; Chung, H.Y.; Koltai, E.; Taylor, A.W.; Goto, S. Exercise, oxidative stress and hormesis. Ageing Res. Rev. 2008, 7, 34-42. [CrossRef]

18. Oguma, Y.; Sesso, H.D.; Paffenbarger, R.S., Jr.; Lee, I.-M. Physical activity and all cause mortality in women: A review of the evidence. Br. J. Sports Med. 2002, 36, 162-172. [CrossRef]

19. Crespo, C.J.; Palmieri, M.R.; Perdomo, R.P.; McGee, D.L.; Smit, E.; Sempos, C.T.; Min, I.; Sorlie, P.D. The Relationship of Physical Activity and Body Weight with All-Cause Mortality Results from The Puerto Rico Heart Health Program. Ann. Epidemiol. 2002, 12, 543-552. [CrossRef]

20. Blair, S.N.; Cheng, Y.; Holder, J.S. Is physical activity or physical fitness more important in defining health benefits? Med. Sci. Sports Exerc. 2001, 33, S379-S399. [CrossRef] [PubMed]

21. Jackson, M.J.; Edwards, R.H.; Symons, M.C. Electron spin resonance studies of intact mammalian skeletal muscle. Biochim. Biophys. Acta Bioenergy 1985, 847, 185-190. [CrossRef]

22. Alessio, H.M.; Goldfarb, A.H.; Cutler, R.G. MDA content increases in fast- and slow-twitch skeletal muscle with intensity of exercise in a rat. Am. J. Physiol. Physiol. 1988, 255, C874-C877. [CrossRef] [PubMed]

23. Castrogiovanni, P.; Imbesi, R. Oxidative stress and skeletal muscle in exercise. Ital. J. Anat. Embryol. 2012, 117, 107-117. [PubMed]

24. Duthie, G.G.; Robertson, J.D.; Maughan, R.J.; Morrice, P.C. Blood antioxidant status and erythrocyte lipid peroxidation following distance running. Arch. Biochem. Biophys. 1990, 282, 78-83. [CrossRef]

25. Reid, M.B.; Haack, K.E.; Franchek, K.M.; Valberg, P.A.; Kobzik, L.; West, M.S. Reactive oxygen in skeletal muscle. I. Intracellular oxidant kinetics and fatigue in vitro. J. Appl. Physiol. 1992, 73, 1797-1804. [CrossRef]

26. Kanter, M. Free radicals, exercise and antioxidant supplementation. Proc. Nutr. Soc. 1998, 57, 9-13. [CrossRef] [PubMed]

27. Ji, L.L.; Radak, Z.; Goto, S. Hormesis and Exercise: How the Cell Copes with Oxidative Stress. Am. J. Pharmacol. Toxicol. 2008, 3, 44-58. [CrossRef]

28. Radak, Z.; Taylor, A.W.; Ohno, H.; Goto, S. Adaptation to exercise-induced oxidative stress: From muscle to brain. Exerc. Immunol. Rev. 2001, 7, 90-107.

29. Gibala, M.J. High-intensity interval training: A time-efficient strategy for health promotion? Curr. Sports Med. Rep. 2007, 6, 211-213. [CrossRef]

30. Hood, M.S.; Little, J.P.; Tarnopolsky, M.A.; Myslik, F.; Gibala, M.J. Low-Volume Interval Training Improves Muscle Oxidative Capacity in Sedentary Adults. Med. Sci. Sports Exerc. 2011, 43, 1849-1856. [CrossRef]

31. Burgomaster, K.A.; Howarth, K.R.; Phillips, S.M.; Rakobowchuck, M.; MacDonald, M.J.; McGee, S.L.; Gibala, M.J. Similar metabolic adaptations during exercise after low volume sprint interval and traditional endurance training in humans. J. Physiol. 2008, 586, 151-160. [CrossRef]

32. Gibala, M.J.; McGee, S.L. Metabolic Adaptations to Short-term High-Intensity Interval Training A Little Pain for a Lot of Gain? Exerc. Sport Sci. Rev. 2008, 36, 58-63. [CrossRef]

33. Downs, S.H.; Black, N. The feasibility of creating a checklist for the assessment of the methodological quality both of randomised and non-randomised studies of health care interventions. J. Epidemiol. Community Health 1998, 52, 377-384. [CrossRef]

34. Ruiz, J.R.; Castro-Piñero, J.; Artero, E.G.; Ortega, F.B.; Sjostrom, M.; Suni, J.; Castillo, M.J. Predictive validity of health-related fitness in youth: A systematic review. Br. J. Sports Med. 2009, 43, 909-923. [CrossRef]

35. Bouzid, M.A.; Hammouda, O.; Matran, R.; Robin, S.; Fabre, C. Changes in Oxidative Stress Markers and Biological Markers of Muscle Injury with Aging at Rest and in Response to an Exhaustive Exercise. PLoS ONE 2014, 9, e90420. [CrossRef]

36. Falone, S.; Mirabilio, A.; Pennelli, A.; Cacchio, M.; Di Baldassarre, A.; Gallina, S.; Passerini, A.; Amicarelli, F. Differential impact of acute bout of exercise on redox- and oxidative damage-related profiles between untrained subjects and amateur runners. Physiol. Res. 2010, 59, 953-961. [CrossRef]

37. Seifi-Skishahr, F.; Siahkohian, M.; Nakhostin-Roohi, B. Influence of aerobic exercise at high and moderate intensities on lipid peroxidation in untrained men. J. Sports Med. Phys. Fit. 2008, 48, 515-521.

38. Kyparos, A.; Salonikidis, K.; Nikolaidis, M.G.; Kouretas, D. Short duration exhaustive aerobic exercise induces oxidative stress: A novel play-oriented volitional fatigue test. J. Sports Med. Phys. Fit. 2007, 47, 483-490.

39. Berzosa, C.; Cebrián, I.; Fuentes-Broto, L.; Gómez-Trullén, E.; Piedrafita, E.; Martínez-Ballarín, E.; López-Pingarrón, L.; Reiter, R.J.; García, J.J. Acute Exercise Increases Plasma Total Antioxidant Status and Antioxidant Enzyme Activities in Untrained Men. J. Biomed. Biotechnol. 2011, 2011, 540458. [CrossRef] [PubMed]

40. Djordjevic, D.Z.; Cubrilo, D.G.; Barudzic, N.S.; Vuletic, M.S.; Zivkovic, V.I.; Nesic, M.; Radovanovic, D.; Djuric, D.M.; Jakovljevic, V.L. Comparison of blood pro/antioxidant levels before and after acute exercise in athletes and non-athletes. Gen. Physiol. Biophys. 2012, 31, 211-219. [CrossRef] [PubMed]

41. Finkler, M.; Hochman, A.; Pinchuk, I.; Lichtenberg, D. In Healthy Young Men, a Short Exhaustive Exercise Alters the Oxidative Stress Only Slightly, Independent of the Actual Fitness. Oxidative Med. Cell. Longev. 2016, 2016, 9107210. [CrossRef]

42. Jammes, Y.; Steinberg, J.G.; Brégeon, F.; Delliaux, S. The oxidative stress in response to routine incremental cycling exercise in healthy sedentary subjects. Respir. Physiol. Neurobiol. 2004, 144, 81-90. [CrossRef] [PubMed]

43. Miyazaki, H.; Oh-Ishi, S.; Ookawara, T.; Kizaki, T.; Toshinai, K.; Ha, S.; Haga, S.; Ji, L.L.; Ohno, H. Strenuous endurance training in humans reduces oxidative stress following exhausting exercise. Eur. J. Appl. Physiol. 2001, 84, 1-6. [CrossRef]

44. Parker, L.; McGuckin, T.A.; Leicht, A.S. Influence of exercise intensity on systemic oxidative stress and antioxidant capacity. Clin. Physiol. Funct. Imaging 2014, 34, 377-383. [CrossRef] [PubMed] 
45. Steinberg, J.G.; Ba, A.; Brégeon, F.; Delliaux, S.; Jammes, Y. Cytokine and Oxidative Responses to Maximal Cycling Exercise in Sedentary Subjects. Med. Sci. Sports Exerc. 2007, 39, 964-968. [CrossRef]

46. Ammar, A.; Trabelsi, K.; Boukhris, O.; Glenn, J.M.; Bott, N.; Masmoudi, L.; Hakim, A.; Chtourou, H.; Driss, T.; Hoekelmann, A.; et al. Effects of Aerobic-, Anaerobic- and Combined-Based Exercises on Plasma Oxidative Stress Biomarkers in Healthy Untrained Young Adults. Int. J. Environ. Res. Public Health 2020, 17, 2601. [CrossRef] [PubMed]

47. Baker, J.S.; Bailey, D.M.; Hullin, D.; Young, I.; Davies, B. Metabolic implications of resistive force selection for oxidative stress and markers of muscle damage during $30 \mathrm{~s}$ of high-intensity exercise. Eur. J. Appl. Physiol. 2004, 92, 321-327. [CrossRef]

48. Groussard, C.; Rannou-Bekono, F.; Machefer, G.; Chevanne, M.; Vincent, S.; Sergent, O.; Cillard, J.; Gratas-Delamarche, A. Changes in blood lipid peroxidation markers and antioxidants after a single sprint anaerobic exercise. Eur. J. Appl. Physiol. 2003, 89, 14-20. [CrossRef]

49. Wiecek, M.; Szymura, J.; Maciejczyk, M.; Kantorowicz, M.; Szygula, Z. Anaerobic Exercise-Induced Activation of Antioxidant Enzymes in the Blood of Women and Men. Front. Physiol. 2018, 9, 9. [CrossRef]

50. Bogdanis, G.C.; Stavrinou, P.; Fatouros, I.G.; Philippou, A.; Chatzinikolaou, A.; Draganidis, D.; Ermidis, G.; Maridaki, M. Short-term high-intensity interval exercise training attenuates oxidative stress responses and improves antioxidant status in healthy humans. Food Chem. Toxicol. 2013, 61, 171-177. [CrossRef] [PubMed]

51. Fisher, G.; Schwartz, D.D.; Quindry, J.; Barberio, M.D.; Foster, E.B.; Jones, K.W.; Pascoe, D.D. Lymphocyte enzymatic antioxidant responses to oxidative stress following high-intensity interval exercise. J. Appl. Physiol. 2011, 110, 730-737. [CrossRef]

52. Maleki, B.H.; Tartibian, B.; Chehrazi, M. The effects of three different exercise modalities on markers of male reproduction in healthy subjects: A randomized controlled trial. Reproduction 2017, 153, 157-174. [CrossRef]

53. Jamurtas, A.Z.; Fatouros, I.G.; Deli, C.K.; Georgakouli, K.; Poulios, A.; Draganidis, D.; Papanikolaou, K.; Tsimeas, P.; Chatzinikolaou, A.; Avloniti, A.; et al. The Effects of Acute Low-Volume HIIT and Aerobic Exercise on Leukocyte Count and Redox Status. J. Sports Sci. Med. 2018, 17, 501-508.

54. Parker, L.; Trewin, A.J.; Levinger, I.; Shaw, C.S.; Stepto, N.K. Exercise-intensity dependent alterations in plasma redox status do not reflect skeletal muscle redox-sensitive protein signaling. J. Sci. Med. Sport 2018, 21, 416-421. [CrossRef] [PubMed]

55. Wadley, A.J.; Chen, Y.-W.; Lip, G.Y.; Fisher, J.P.; Aldred, S. Low volume-high intensity interval exercise elicits antioxidant and anti-inflammatory effects in humans. J. Sports Sci. 2016, 34, 1-9. [CrossRef]

56. Kröpfl, J.M.; Beltrami, F.G.; Rehm, M.; Gruber, H.-J.; Stelzer, I.; Spengler, C.M. Acute exercise-induced glycocalyx shedding does not differ between exercise modalities, but is associated with total antioxidative capacity. J. Sci. Med. Sport 2021, 24, 689-695. [CrossRef]

57. Fogarty, M.C.; Hughes, C.M.; Burke, G.; Brown, J.C.; Trinick, T.R.; Duly, E.; Bailey, D.M.; Davison, G.W. Exercise-induced lipid peroxidation: Implications for deoxyribonucleic acid damage and systemic free radical generation. Environ. Mol. Mutagen. 2011, 52, 35-42. [CrossRef] [PubMed]

58. Tryfidou, D.V.; McClean, C.; Nikolaidis, M.G.; Davison, G.W. DNA Damage Following Acute Aerobic Exercise: A Systematic Review and Meta-analysis. Sports Med. 2020, 50, 103-127. [CrossRef]

59. Cipryan, L. IL-6, Antioxidant Capacity and Muscle Damage Markers Following High-Intensity Interval Training Protocols. J. Hum. Kinet. 2017, 56, 139-148. [CrossRef]

60. Cipryan, L. The effect of fitness level on cardiac autonomic regulation, IL-6, total antioxidant capacity, and muscle damage responses to a single bout of high-intensity interval training. J. Sport Health Sci. 2018, 7, 363-371. [CrossRef] [PubMed]

61. Turner, J.E.; Bosch, J.A.; Drayson, M.T.; Aldred, S. Assessment of oxidative stress in lymphocytes with exercise. J. Appl. Physiol. 2011, 111, 206-211. [CrossRef] [PubMed]

62. Farney, T.M.; Mccarthy, C.G.; Canale, R.E.; Schilling, B.K.; Whitehead, P.N.; Bloomer, R.J. Absence of Blood Oxidative Stress in Trained Men after Strenuous Exercise. Med. Sci. Sports Exerc. 2012, 44, 1855-1863. [CrossRef] [PubMed]

63. El Abed, K.; Rebai, H.; Bloomer, R.J.; Trabelsi, K.; Masmoudi, L.; Zbidi, A.; Sahnoun, Z.; Hakim, A.; Tabka, Z. Antioxidant Status and Oxidative Stress at Rest and in Response to Acute Exercise in Judokas and Sedentary Men. J. Strength Cond. Res. 2011, 25, 2400-2409. [CrossRef]

64. Otocka-Kmiecik, A.; Lewandowski, M.; Stolarek, R.; Szkudlarek, U.; Nowak, D.; Orlowska-Majdak, M. Effect of single bout of maximal excercise on plasma antioxidant status and paraoxonase activity in young sportsmen. Redox Rep. 2010, 15, $275-281$. [CrossRef] [PubMed]

65. Otocka-Kmiecik, A.; Lewandowski, M.; Szkudlarek, U.; Nowak, D.; Orlowska-Majdak, M. Aerobic Training Modulates the Effects of Exercise-Induced Oxidative Stress on PON1 Activity: A Preliminary Study. Sci. World J. 2014, 2014, 230271. [CrossRef]

66. Suzuki, K.; Nakaji, S.; Yamada, M.; Liu, Q.; Kurakake, S.; Okamura, N.; Kumae, T.; Umeda, T.; Sugawara, K. Impact of a Competitive Marathon Race on Systemic Cytokine and Neutrophil Responses. Med. Sci. Sports Exerc. 2003, 35, 348-355. [CrossRef] [PubMed]

67. Chaki, B.; Pal, S.; Chattopadhyay, S.; Bandyopadhyay, A. High-intensity exercise-induced oxidative stress in sedentary prepubertal \& post-pubertal boys: A comparative study. Indian J. Med. Res. 2019, 150, 167-174. [CrossRef]

68. Sureda, A.; Tauler, P.; Aguiló, A.; Cases, N.; Fuentespina, E.; Córdova, A.; Tur, J.A.; Pons, A. Relation between oxidative stress markers and antioxidant endogenous defences during exhaustive exercise. Free Radic. Res. 2005, 39, 1317-1324. [CrossRef]

69. Margaritis, I.; Tessier, F.; Richard, M.-J.; Marconnet, P. No Evidence of Oxidative Stress after a Triathlon Race in Highly Trained Competitors. Int. J. Sports Med. 1997, 18, 186-190. [CrossRef] 
70. Rokitzki, L.; Logemann, E.; Sagredos, A.N.; Murphy, M.; Wetzel-Roth, W.; Keul, J. Lipid peroxidation and antioxidative vitamins under extreme endurance stress. Acta Physiol. Scand. 1994, 151, 149-158. [CrossRef]

71. Leaf, D.A.; Kleinman, M.T.; Hamilton, M.; Barstow, T.J. The effect of exercise intensity on lipid peroxidation. Med. Sci. Sports Exerc. 1997, 29, 1036-1039. [CrossRef] [PubMed]

72. Ugras, A.F. Effect of high intensity interval training on elite athletes' antioxidant status. Sci. Sports 2013, 28, 253-259. [CrossRef]

73. Asami, S.; Hirano, T.; Yamaguchi, R.; Itoh, H.; Kasai, H. Reduction of 8-hydroxyguanine in human leukocyte DNA by physical exercise. Free Radic. Res. 1998, 29, 581-584. [CrossRef]

74. Shing, C.M.; Peake, J.M.; Ahern, S.M.; Strobel, N.A.; Wilson, G.; Jenkins, D.G.; Coombes, J.S. The effect of consecutive days of exercise on markers of oxidative stress. Appl. Physiol. Nutr. Metab. 2007, 32, 677-685. [CrossRef] [PubMed]

75. Boisseau, N.; Delamarche, P. Metabolic and Hormonal Responses to Exercise in Children and Adolescents. Sports Med. 2000, 30, 405-422. [CrossRef] 